\title{
Le développement de l'industrie du nickel et la transformation de la valeur environnementale en Nouvelle- Calédonie
}

\author{
Matthias Kowasch ${ }^{1}$ \\ Institut de Recherche pour le Développement, New Caledonia
}

\section{Introduction}

Pour J. Bonnemaison, la représentation d'un territoire, d'un espace, d'une réalité est plus importante que la réalité même: «La représentation que les sociétés où les individus se font d'une réalité précise, d'un être géographique, est plus importante que la réalité même; ou plus exactement que cette réalité n'existe que dans la représentation que l'on se fait d'elle » (Bonnemaison 2000: 51).

La Nouvelle-Calédonie, un pays d'outre-mer français d'une superficie d'environ $19,000 \mathrm{~km}^{2}$ (7,335 square miles), à quelques $1,500 \mathrm{~km}$ (932 miles) à l'est de la côte australienne, se caractérise par une grande diversité culturelle (Gay 2003; Doumenge 2000). Selon le recensement de 2009, la population autochtone, les Kanak, représentent environ $40 \%$ de la population totale, les Européens $29 \%$, les Wallisiens et Futuniens 9\%, les Asiatiques (principalement Vietnamiens et Indonésiens) 4\%, les Tahitiens $2 \%$ et les $\mathrm{Ni}$-Vanuatais $1 \%$. Cependant, $8 \%$ se déclarent d'appartenir à plusieurs communautés, et $1 \%$ n'était pas déclaré (http://www.isee.nc). Le mode de vie de la communauté kanak se différencie toujours du mode de vie de la communauté d'origine européenne: on peut parler d'une cohabitation d'une société agraire multiséculaire océanienne démographiquement minoritaire, et d'une société majoritairement urbaine, cosmopolite, à dominante européenne, largement métissée (environ $60 \%$ de la population totale). La représentation que les Kanak se font de l'espace est déterminante pour la construction de leur identité.

Le présent travail étudiera en première partie la construction de l'identité kanak et son inscription dans l'espace. La valeur que les populations accordent à leur territoire, à leur environnement sera comparée avec d'autres modèles de valeur. L'identité n'est pas quelque chose de figée, elle représente un processus de construction et de reconstruction permanente, ce qui a déjà été constaté par S. Speed (2002: 222). Le présent article placera le « modèle kanak » dans un contexte plus large. Après une description de l'importance de l'industrie du nickel en Nouvelle-Calédonie et des impacts socio-économiques dus aux exploitations minières et à la mise en place de nouvelles usines de traitement, j'analyserai les transformations de la valeur environnementale et les mutations foncières que l'industrie minière entraîne. La volonté de bénéficier financièrement des exploitations minières semble être la raison pour de nombreuses demandes de royalties, mais également des revendications foncières. Trois études de cas démontreront les enjeux autour de la participation aux projets miniers. Ainsi seront présentées les tribus de Bako et d'Oundjo à proximité du site industriel de Vavouto où est actuellement construite une nouvelle usine de traitement de nickel. Ensuite sera analysée une fédération coutumière qui à la base revendiquait des terres, mais qui travaille actuellement en tant qu'entreprise de sous-traitance pour l'opérateur industriel.

Le géographe N. Castree voit dans les revendications foncières des peuples autochtones un moyen de « reappropriation de ressources » :

Proprietary demands are at the extreme end of the spectrum of claims that indigenous peoples can and do make about themselves and their aspirations ... I rehearse some of the arguments that have been made in favour of indigenous proprietary reappropriation of 'their' resources (Castree 2004: 160).

Le contexte socio-politique des revendications foncières en Nouvelle-Calédonie sera analysé avant que les arguments de Castree seront confrontés à d'autres auteurs ayant travaillé sur des questions foncières: Michael Watts, Doreen Massey, David Harvey et Pierre-Yves Le Meur.

\section{Construction d'identité et transformations de valeur}

Comme dans d'autres sociétés traditionnelles agraires, l'identité collective kanak se construit sur l'histoire du groupe inscrit dans un environnement contrôlé et valorisé de longue date: la profondeur du temps est toujours source de respect, voire de prestige. Pour la population kanak, le lien à la terre est très important et le territoire est un espace culturel d'identification. Avant l'arrivée des Européens, la terre n'était nullement l'objet de transactions marchandes, mais pouvait faire l'objet d'échanges conventionnés

\footnotetext{
${ }^{1}$ Dr. Matthias Kowasch, Institut de Recherche pour le Développement (IRD), UMR Espace-Dev, Nouvelle Calédonie et l'Université de la Nouvelle-Calédonie. Matthiaskowasch "at" googlemail.com. Je tiens à remercier l'équipe de l'UMR Espace-DEV qui m'a accueilli pendant mon terrain de thèse de doctorat et après, m'a apporté un soutien technique et m'a toujours encouragé.
} 
entre groupes lignagers (enjeu foncier), voire entre alliances claniques (enjeu politique). Comme ailleurs dans le monde, la propriété d'un terrain procède de la constitution d'un territoire politique englobant au sein d'un espace naturel: en milieu kanak, ce territoire se définit par ses limites, ses allées résidentielles, ses tertres de case et ses espaces de culture (Naepels 2006: 49). L'appropriation d'un espace résulte d'un travail de fondation et de nomination. Les véritables " propriétaires » d'un lieu sont donc les fondateurs, ceux qui le défrichèrent au plus profond du temps, « ceux qui en premiers ont coupés les racines des plantes sauvages » (réflexion faite par J.M. Tjibaou à J.-P. Doumenge en 1976). Pour Donna Winslow dans cette revue (1995:5), « le plan de référence du clan était le tertre fondé par le clan ancêtre ». Ce qui fait la spécificité du clan ou du lignage, c'est d'abord son histoire: le lieu et les circonstances de son apparition, le parcours qu'il effectua, la symbolique qui lui est reconnue. Cette histoire est racontée et transmise lors des cérémonies coutumières telle qu'un mariage ou un deuil. L'identité sociale et la légitimité de tout groupe nominatif se présente alors comme un itinéraire, comme une suite de lieux où le groupe est passé (Naepels 2006: 50). Cet itinéraire confirme la thèse que l'identité n'est jamais donnée, mais toujours construite. Cela vaut pour les Kanak ainsi que pour toute autre population. Ainsi, Castells (1997: 7) note: «It is easy to agree on the fact that ... all identities are constructed. The real is how, from what, by whom and for what. " Pour les Kanak, la construction de leur identité s'inscrit dans l'espace, sur la base de l'itinéraire clanique. Mais ce parcours clanique ne semble pas être particulière à la l'identité kanak, car d'autres peuples autochtones réclament également « a particular historical (and geographical) trajectory of their own » (Dirlik, 1996: 18). Ainsi, les populations autochtones se réfèrent à une trajectoire historique qui est tracée dans l'espace et qui est en perpétuelle réactualisation (Naepels 1998; Le Meur 2010b). La propriété kanak n'est non seulement suspendue à une constante réactualisation, parce que le parcours du clan peut évoluer, mais aussi parce qu'une terre peut être donnée. Le fondateur n'est alors pas le seul à pouvoir revendiquer un terrain. Suivant une « dynamique d'accueil », le nouvel arrivant reçoit du fondateur un terrain qu'il pourra habiter et cultiver. A partir du moment où la terre est cédée, une terre se transmet au sein du groupe agnatique de l'arrivant sans que le donateur puisse la récupérer ou intervenir sur l'usage qui en est fait. Néanmoins, cette cession est liée à des conditions générales de l'accueil: que les descendants de l'accueilli repartent et l'accueillant récupère le bien concédé pour le réaffecter. Tout don de terre est mémorisé par les générations successives et fait annuellement l'objet d'un " don de première récolte (prémice) » du groupe de l'accueilli à celui de l'accueillant. Ce don s'inscrit dans l'ensemble des relations qui unissent le clan de l'accueillant et le clan du nouveau propriétaire (Naepels 2006: 49). Dans tous les cas, l'arrivant doit garder en mémoire les échanges qui ont sanctionné son installation.

Dans ce contexte, il est important de noter que cette cession d'une terre définissait moins des transferts de propriété que la superposition de différentes légitimités: la propriété n'est pas univoque, mais consiste plutôt en des titres concurrents. Le fondateur garde ainsi un lien particulier à la terre qu'il fonda. L'accueilli de son côté peut faire valoir le fait qu'il y ait résidé, qu'il ait effectué des échanges avec le fondateur et qu'il y ait travaillé, mais il ne peut dans aucun cas contester le fait d'avoir reçu la terre d'un autre.

L'environnement n'a donc non seulement une valeur d'usage pour la société kanak, mais également une valeur patrimoniale où tous les objets environnementaux possèdent une certaine valeur. Des travaux sur l'économie de l'environnement furent déjà réalisés par R. Constanza et al. (1997), par Moberg et Folke (1999) et par G. David et al. (2007), Les auteurs tentèrent de donner une valeur aux écosystèmes. Le modèle ci-dessous (Figure 1) représente un outil très simple qui permet de démontrer la transformation de la valeur accordée à l'environnement.

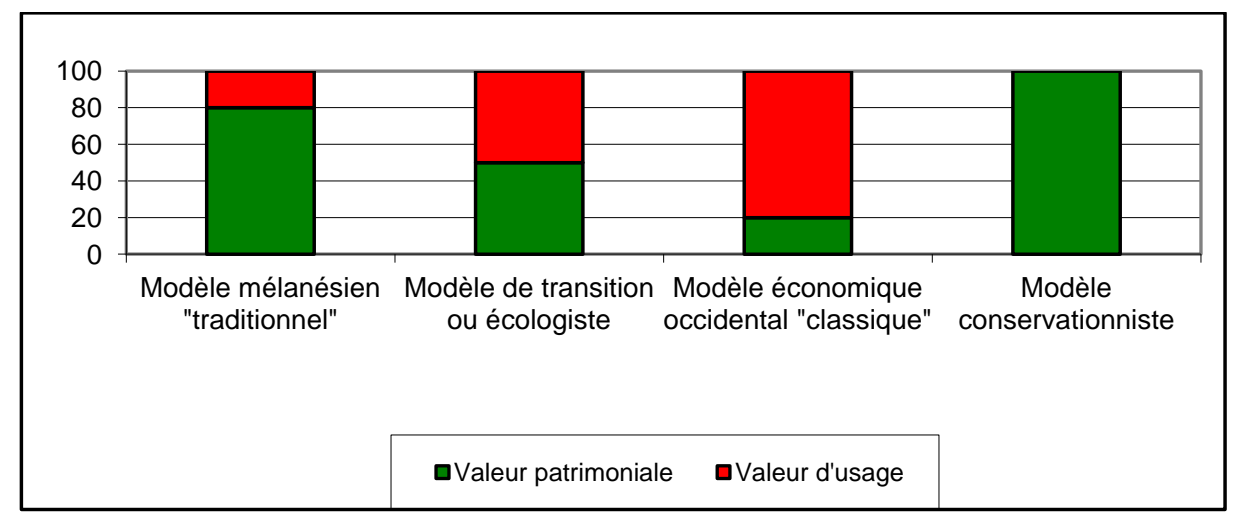

Figure 1 : Importance relative accordée à la valeur d'usage et à la valeur patrimoniale par les acteurs selon des modèles simplifiés. Source: Communication personnelle de J.-B. Herrenschmidt, 2010.

La valeur patrimoniale se transcrit principalement dans les lieux toponymiques qui représentent un réservoir culturel essentiel qu'on ne peut comprendre qu'en maîtrisant la langue vernaculaire du clan fondateur de l'endroit. En 1995, D. Winslow remarque que « chaque nom de clan représente un toponyme 
- le nom d'un site où l'ancêtre du clan s'est manifesté ainsi que tous les sites successifs occupés dans l'histoire du clan » (Winslow 1995: 14). Les ancêtres sont en effet omniprésents et leur présence est ressentie dans le passage d'un papillon, dans la pluie qui tombe ou dans l'eau d'une rivière qui tourbillonne. La perception de la population kanak s'oriente au modèle mélanésien « traditionnel » dans lequel la valeur patrimoniale est très nettement supérieure à la valeur d'usage (Figure 1). Le modèle porte un discours culturel fort liant les lieux à un mythe, une histoire spécifique, des symboles, à des savoirs traditionnels. A des pratiques culturelles comprenant aussi la pêche ou l'agriculture vivrière s'ajoute l'usage étant décrit comme la ponction de ce qui est nécessaire à vivre et à se reproduire (Kowasch 2010: 52). Une plus grande implication dans le salariat explique partiellement la perte de ce réservoir culturel et la transformation du modèle mélanésien «traditionnel »: les jeunes qui travaillent ont moins de temps et s'intéressent moins aux savoirs des ancêtres. En perdant ce savoir historico-mythique, la valeur patrimoniale d'une terre se trouve considérablement dépréciée (Kowasch 2010: 477). La perception se transforme alors en un modèle de transition ou écologiste. Dans ce modèle, le discours cherche à équilibrer l'importance des valeurs d'usage et patrimoniale, soit décrivant une évolution des modes de vie mélanésiens entrés dans l'économie monétaire, soit décrivant au contraire une évolution du discours récente du monde occidental sur le développement durable qui cherche à introduire une notion d'équilibre entre usage et capacité du milieu à garder ses caractéristiques patrimoniales.

Selon le modèle économique occidental « classique », la valeur socio-économique d'un milieu est souvent réduite à sa valeur d'usage (par exemple la quantité pêchée sur les récifs coralliens pour l'économie monétaire ou vivrière des acteurs locaux), c'est-à-dire qu'elle est limitée aux biens et services économiques que l'environnement assure, n'accordant peu de place à des références culturelles ou territoriales. Le modèle « conservationniste » de son côté évacue la question de l'usage en prônant la mise en réserve d'espaces naturels perçus comme patrimoine commun. Seule la valeur patrimoniale y existe.

Tous les sites sacrés possèdent une certaine valeur patrimoniale. Compte tenu que des lieux à valeur patrimoniale existent sur site minier et sur les terres où seront installées des zones d'activités pour l'installation d'infrastructures nécessaires pour une usine métallurgique, il faudra se poser la question suivante: la valeur de la terre en question est-elle en train d'être transformée par les acteurs " propriétaires », parce qu'il existe un projet minier ou métallurgique? Quels sont les acteurs, et quels conflits engendre ce remodelage du territoire? Les enjeux et "l'arène sociale » de ces mutations territoriales seront ainsi à analyser. A. Amin (2002: 397) ne parle dans ce contexte pas de «politics in place », mais de « politics of place ». Quels sont alors les enjeux de cette « politique de l'endroit »?

\section{Méthode}

Afin de répondre à ces questions, j'ai effectué des terrains pendant une durée totale d'environ 1 an dans la zone Voh-Koné-Pouembout en province Nord de la Nouvelle-Calédonie, mais aussi à la commune de Yaté en province Sud. Les études de cas de cet article se baseront sur les enquêtes réalisées dans les tribus de Baco (commune de Koné) et d'Oundjo (commune de Voh). Les enquêtes quantitatives auprès des ménages tribaux sont croisées et complétées par des enquêtes de type qualitatives avec des personnes clés.

Pour connaître la valeur de l'environnement, il fallait cartographier les lieux toponymiques à valeur patrimoniale touchés par un projet minier ou métallurgique. En d'autres termes, l'enjeu était de développer une méthode d'analyse spatiale permettant de cartographier la valeur patrimoniale de l'espace attribuée par les populations locales. Cette spatialisation fut réalisée à l'aide d'un GPS et du programme ArcGIS. Les personnes ressources notèrent les noms des lieux dans leur langue (haeke, haveke ou bwatoo) dans un cahier sur le terrain ou simplement sur une carte topographique en papier; ces noms furent ensuite importés dans ArcGIS.

Les lieux toponymiques renvoient aux interactions entre différents acteurs. Tandis qu'un endroit peut avoir une certaine signification pour un groupe, il n'a aucune valeur pour un autre. Différentes significations, importances et légitimités peuvent se superposer. Ainsi, les différentes logiques d'acteurs seront à analyser. Cette approche permet de poser la question de l'action collective en liaison directe avec les stratégies individuelles. Ces stratégies impliquent les notions de conflit, d'arrangement et de consensus. Les conflits doivent être insérés dans une chaîne d'événements et de processus sociaux. Dans le cas où différentes légitimités foncières se superposent sur une même terre, plusieurs cartes doivent être réalisées, une carte avec chaque clan pouvant faire valoir des " droits à l'endroit ». Les différents acteurs de l'endroit et leurs enjeux sont à analyser et décortiquer.

\section{L'industrie du nickel et les disparités démo-spatiales en Nouvelle-Calédonie}

Jusqu'à présent, la Nouvelle-Calédonie profite largement des transferts financiers de l'Etat français; l'effort financier en faveur de l'outre-mer continue à s'accroître. Mais les acteurs économiques locaux qui ont la chance de profiter de l'injection massive des fonds publics métropolitains placent leurs bénéfices souvent en métropole, en Union Européenne, aux Etats-Unis ou en Australie. Ils ne les réinvestissent pas sur le "Caillou », perçu plus comme «espace de prédation » immédiate que comme " espace de construction à long terme ». Ils n'ont pas confiance dans l'avenir du pays ou bien, ils pensent que l'argent est plus facile à gagner à l'extérieur. Les transferts publics entrant dans le territoire engendrent un flux inverse égal, correspondant à un supplément d'importations et au placement à l'extérieur de l'épargne non 
investie sur le territoire. L'économie de la Nouvelle-Calédonie peut être décrite comme une « économie assistée » (Freyss 1995; Perret 2002), ce qui entraîne un déficit chronique de sa balance commerciale et qui n'est pas orientée vers l'effort productif, mais vers la captation d'une rente. Les exportations de la Nouvelle-Calédonie dépendent largement du prix du nickel sur le marché mondial, car elles représentent environ 95\% de la valeur totale des exportations. Cette large dépendance du secteur minier a des effets négatifs sur la diversification de l'économie calédonienne. G. Bridge (2004: 228) constate que l'industrie minière freine généralement le développement des autres secteurs économiques: « (...) mineral booms negatively affect the performance of other export sectors via a form of 'medium term deindustrialization' ».

Le nickel est le moteur du développement économique et le premier produit d'exportation de la Nouvelle-Calédonie. Environ $50 \%$ de la surface de ressource minière identifiée ne furent pas encore suffisamment explorés. Tandis que l'archipel possède $25 \%$ des ressources minérales en nickel inventoriées dans le monde, la seule usine de traitement («Doniambo » à Nouméa) ne produit que 5,5\% du nickel-métal à l'échelle de la planète. La «Société Le Nickel » (SLN), une filiale du groupe français Eramet, dirige cette usine: elle est jusqu'à présent le seul producteur de nickel-métal dans le pays. Du point de vue économique, le souhait de traiter localement une plus grande partie du minerai brut s'explique: cela dégagerait une plus grande valeur ajoutée et permettrait de donner de l'emploi à une population souffrant de chômage, particulièrement pour la main-d'œuvre peu ou pas spécialisée. Depuis longtemps existe donc l'idée de construire une deuxième usine de traitement du nickel en NouvelleCalédonie. Aujourd'hui, le groupe canado-brésilien Vale Inco d'une part et KNS (« Koniambo Nickel SAS »), un consortium des Suisses de Xstrata et de la SMSP ("Société Minière du Sud Pacifique ») locale, d'autre part mettent à exécution cette idée. Elles sont en train de construire deux nouvelles usines de traitement, l'une dans la province Sud (Vale Inco), l'autre dans la province Nord (Xstrata/SMSP) de la "Grande-Terre », l'île principale de la Nouvelle-Calédonie (Image 1). Une entrée en concurrence de projets métallurgiques ne se trouvant pas dans une même région, voire sur un même gisement, mais d'une province à l'autre est un phénomène tout à fait nouveau pour la Nouvelle-Calédonie. Le pays verra donc émerger non seulement une nouvelle usine métallurgique, mais deux. De ce fait, la Nouvelle-Calédonie pourra tripler sa production annuelle de 60,000 tonnes à 180,000 tonnes de nickel-métal.

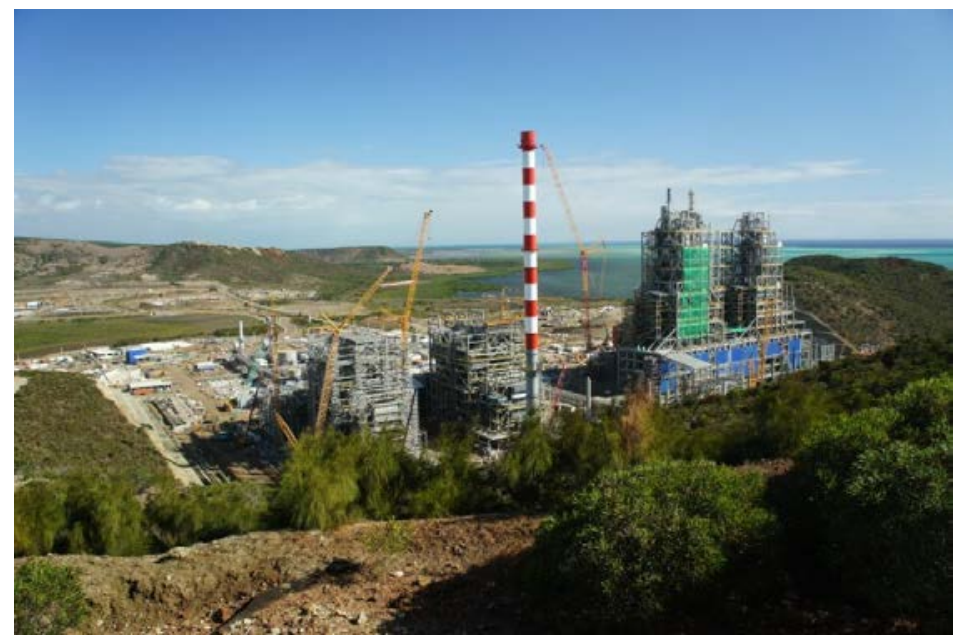

Image 1 : Le chantier de l'usine du Nord en Novembre 2011. Source : M. Kowasch 2011.

De plus, l'implantation d'une usine métallurgique en province Nord devrait contribuer au rééquilibrage du territoire calédonien (Carnuccini et Guillaud 1999: 83-84) qui se caractérise d'un point de vue démographique et économique par une hypertrophie de la ville-capitale Nouméa située en province Sud. Sur une population totale de 245,580 en 2009, 163,723 (soit 66,6\%) habitent dans le Grand Nouméa qui regroupe les communes de Nouméa (97,579 habitants), Mont-Dore (25,683), Dumbéa $(24,103)$ et Païta $(16,358)$. Environ $75 \%$ des employés travaillent à Nouméa, $70 \%$ des employeurs calédoniens y sont présents; 73\% des richesses y sont produites. L'économie du pays est donc dominée par la ville-capitale et ceux qui le représentent le mieux, à savoir les Européens et les Asiatiques (Doumenge 2002, 2000). La « colonisation » de l'archipel calédonien se voit toujours dans la répartition spatiale de la population qui souligne cette dualité des espaces de vie en Nouvelle-Calédonie. Alors que les Kanak représentent respectivement $78 \%$ et $97 \%$ de la population totale dans les provinces « Nord » et « Îles Loyauté », ils sont minoritaires en province Sud où $45 \%$ de la population sont d'origine européenne.

La puissance économique émanant de la communauté européenne s'est ancrée en grande partie dans la mine. La participation marginale de la population kanak à l'économie marchande dans le pays ne contribue pas au développement d'une identité culturelle unifiée. Le territoire se caractérise donc par une fragmentation spatiale problématique. Toutes les communes ne participent pas au même degré au 
développement économique. En 1999, J. Guiart constate un «jeu inégal de deux sociétés et de deux économies non encore réellement intégrées, l'Européenne et la Canaque » (Guiart 1999: 10). Cette intégration marginale de populations autochtones dans l'économie du marché et leur participation faible à l'exploitation minière furent constatées dans d'autres pays anciennement colonisés, on pourrait ainsi citer les Innus et les Inuits au Canada, les Bororos et les Awas au Brésil, les Gwi au Botswana, et les Aborigènes en Australie. Bien souvent, les populations autochtones n'ont guère bénéficié des exploitations des matières premières: tel est aussi le constat de B. Parry (2002): " Meanwhile, indigenous groups from the south Pacific to the Arctic circle are being 'mined' by transnational companies for potentially marketable tangible and intangible resources with little or no compensation offered in return ». La question est alors comment les populations kanak peuvent davantage tirer profit de l'exploitation du nickel dans leur pays? Jusqu'à récemment, leur participation s'est principalement réduite à un emploi en tant que main d'œuvre.

Mais il existe d'autres moyens de participation. En province Nord de la Nouvelle-Calédonie, les autorités politiques, qui sont en majorité issues du mouvement indépendantiste kanak, souhaitent rééquilibrer le territoire en promouvant le développement de l'industrie du nickel (Néaoutyine 2006; Pitoiset et Wéry 2008). La SMSP constitue en quelque sorte le «bras armé » de la province Nord, car elle est une filiale à hauteur de $87 \%$ de la société mixte de développement de la province Nord, la SOFINOR ( «Société de financement et d'investissement de la Province Nord »). La SMSP, qui détient 51\% des actions dans le projet Koniambo, possède donc une valeur symbolique forte pour le mouvement indépendantiste kanak. La mise en service de l'usine est prévue pour 2012; la pleine capacité de production annoncée pour 2013.

La dualité des espaces de vie (Kanak d'une part, Européens d'autre part) se montre également dans la juxtaposition de terres de droit commun et de terres de droit coutumier. Le partage décrit dans l'accord de Nouméa définit juridiquement deux types de terres (ou de propriétés): "Les terres coutumières seront constituées des réserves, des terres attribuées aux " groupements de droit particulier local » et des terres qui seront attribuées au titre du lien à la terre. Il n'y aura ainsi que les terres coutumières et les terres de droit commun " (Point 1.4. du document d'orientation dans l'accord de Nouméa, in Naepels 2006: 43). Dans l'objectif de rééquilibrer le territoire calédonien en faveur de la population kanak, l'État français entama dans les années 1970 une réforme foncière que l'ADRAF (« Agence de développement rural et d'aménagement foncier ») juge "satisfaisant » en 2010, puisque l'équilibre entre le foncier kanak (295,300 ha) et le foncier privé européen (295,300 ha) est devenu une réalité sur la Grande-Terre dans les années 2000-2010 (Tableau 1).

\begin{tabular}{|c|c|c|c|c|c|c|}
\hline Catégories de terres & $\begin{array}{r}1978 \text { (en } \\
\text { ha) }\end{array}$ & $\%$ & $\begin{array}{r}1998 \text { (en } \\
\text { ha) }\end{array}$ & $\%$ & $\begin{array}{r}2010 \text { (en } \\
\text { ha) }\end{array}$ & $\%$ \\
\hline Foncier kanak & 167,788 & 10 & 276,516 & 17 & 295,300 & 18 \\
\hline $\begin{array}{l}\text {-dont réserves autochtones } \\
\text {-dont propriétés claniques } \\
\text {-dont GDPL } \\
\text {-dont propriétés kanak et attributions } \\
\text { individuelles kanak ou sociétaires }{ }^{2}\end{array}$ & 161,788 & & $\begin{array}{r}180,882 \\
8,942 \\
71,692 \\
15,000\end{array}$ & & & \\
\hline Stock ADRAF & & & 24,673 & 1 & 18,200 & 1 \\
\hline Terres privées non kanak ${ }^{3}$ & 402,471 & 25 & 295,851 & 18 & 295,300 & 18 \\
\hline Terrains domaniaux et collectivités ${ }^{4}$ & $1,062,441$ & 65 & $1,035,644$ & 64 & $1,024,100$ & 63 \\
\hline TOTAL Grande Terre $^{5}$ & $1,632,700$ & 100 & $1,632,684$ & 100 & $1,632,900$ & 100 \\
\hline
\end{tabular}

Tableau 1 : Évolution des catégories de foncier entre 1978 et 2010 en Grande-Terre. Source:

ADRAF $2000: 51$.

L'ADRAF, un établissement public d'État ${ }^{6}$, créée en 1986, est autorisée à acquérir, soit à l'amiable, soit à préemption, des terres à vocation agricole, pastorale ou forestière, et à les rétrocéder. L'agence rétrocède les terres à titre gratuit à des GDPL (Groupement de droit particulier local) ou à titre onéreux dans le cadre de projets individuels. Dans la structure des GDPL, les Kanak retrouvent leurs terres

\footnotetext{
${ }^{2}$ Cette catégorie propose une estimation de l'ensemble des propriétés individuelles des Kanak. Le chiffre de 1978 provient d'une estimation fournie par la DTSF (Direction Territoriale des Services Fiscaux) en 1998. Le chiffre de 1998 fut estimé globalement par l'ADRAF par actualisation de l'estimation de 1978 étant entendu que les attributions individuelles effectuées au titre des réformes foncières ne représentent qu'une faible part des acquisitions individuelles des Kanak au cours de la période.

${ }^{3}$ Sauf Belep et Ile des Pins. Le chiffre de 1998 fut obtenu à partir des données de la DTSF fournies en 1998.

${ }^{4}$ Cette catégorie regroupe les terrains domaniaux (Territoire et État), ceux des collectivités locales (communes et provinces) et les terrains urbanisés.

${ }^{5}$ Hors propriétés privées mélanésiennes identifiées et stock ADRAF.

${ }^{6}$ Après le transfert des compétences de l'État à la Nouvelle-Calédonie, prévu par l'accord de Nouméa en 1998, l'ADRAF sera transformée en un établissement public territorial. Le dossier est actuellement en examen.
} 
ancestrales pour un temps indéfini. Les réformes foncières ont encouragé les Kanak à mettre en valeur leurs terres ancestrales et à participer ainsi au développement économique. Malgré cela, l'ADRAF constate un manque de projets économiques sur terres rétrocédées ce qui tient à plusieurs facteurs (ADRAF 2000: 75): Toute création d'entreprise exige un engagement financier (avec un apport personnel et des garanties pour pouvoir contracter un emprunt) ce qui était jusqu'aux années 1980 incompatible avec le statut de droit particulier des ressortissants des tribus - dans les réserves et les terres rétrocédées, la terre étant inaliénable, incommutable, incessible et insaisissable, aucune hypothèque ne pouvait être prise sur le patrimoine foncier. Aujourd'hui, les investisseurs signent avec les autorités coutumières un acte coutumier qui leur donne une certaine garantie foncière, sorte de contrat moral qui peut théoriquement être retiré à tout moment. Les entrepreneurs privés sont donc souvent réservés envers une installation sur terre coutumière. En outre, la remise en état des propriétés coûte cher et les membres du GDPL ne réfléchissent souvent pas au préalable au mode de gestion des terres après l'attribution. De plus, l'organisation sociale des communautés kanak est peu préparée à l'entreprise individuelle en matière agricole ou industrielle. En absence de statuts juridiques, le GDPL ne peut pas être considéré comme une structure économique. A cela s'ajoute les difficultés pour accéder à des financements en l'absence de garanties foncières et un manque de formation. C'est pourquoi, l'ADRAF est aujourd'hui plus occupée avec la gestion et l'aménagement qu'avec la rétrocession de terres. Compte tenu des différentes difficultés, la mise en valeur des terres coutumières de la tribu de Baco en province Nord est souvent décrite comme un « projet phare pour la Nouvelle-Calédonie ». A part un emploi direct chez l'opérateur minier, les Kanak peuvent donc participer au développement de l'industrie du nickel en mettant en valeur leurs terres (avec l'implantation d'une zone industrielle ou de nouveaux logements locatifs par exemple).

\section{Etudes de cas}

L'étude empirique sur le remodelage du territoire porte sur deux tribus en province Nord de la Nouvelle-Calédonie, celles de Baco et d'Oundjo, ainsi que sur une fédération coutumière appelé « Djelawe ». Les deux tribus se trouvent à proximité du site industriel de Vavouto où l'opérateur minier KNS construit la future « usine du Nord » (Figure 2).

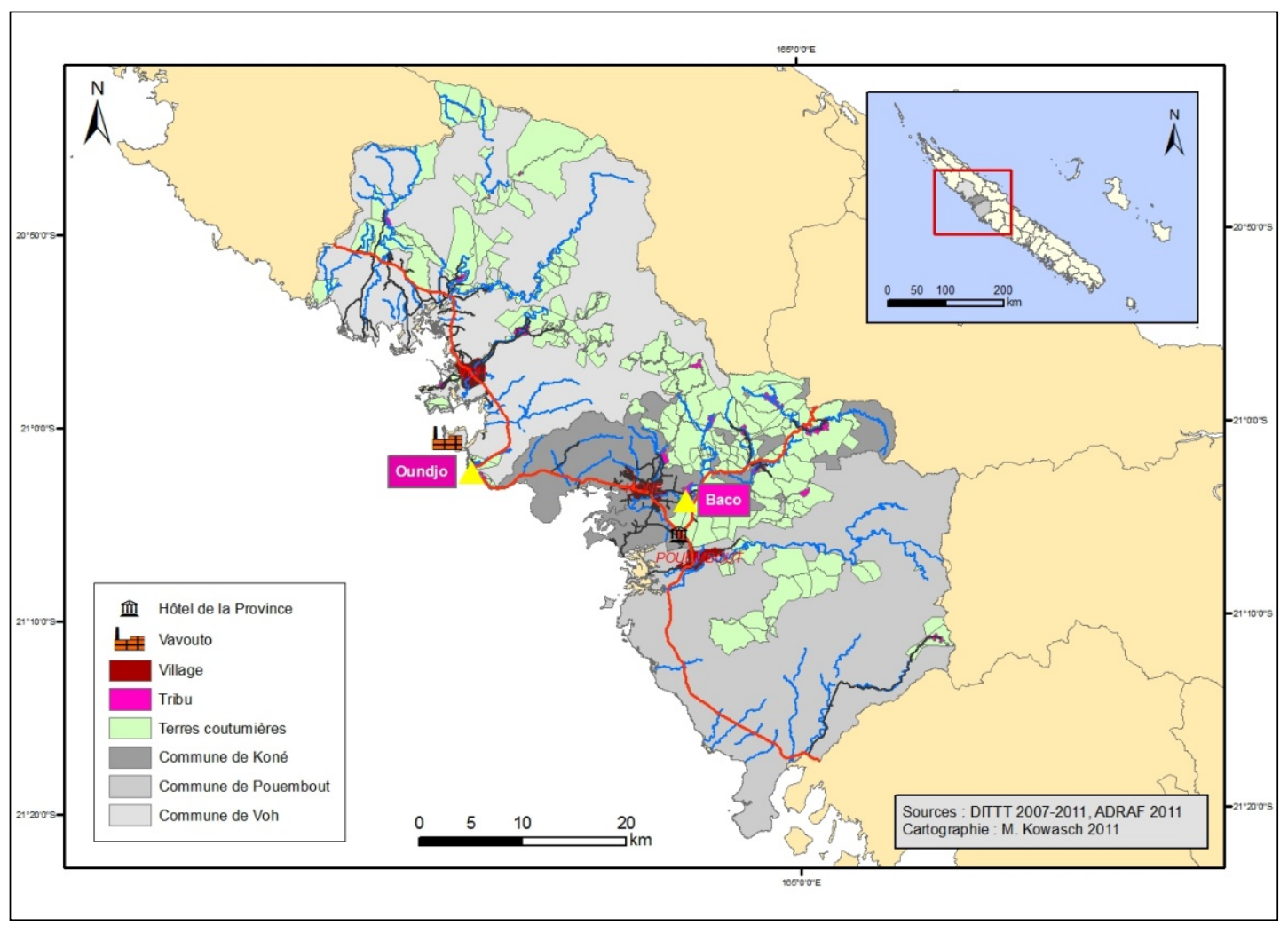

Figure 2 : Situation géographique des tribus d'Oundjo et de Baco. Source: M. Kowasch. 


\section{La tribu d'Oundjo}

La tribu d'Oundjo est la tribu la plus proche du site industriel qui se trouve à une distance d'1,5 km (0.93 miles) (Figure 2). Selon le recensement de 1996, la tribu d'Oundjo compte 301 personnes. Les habitations de la tribu d'Oundjo se trouvent presque entièrement sur les terres de la réserve. L'agrandissement de la réserve se situe juste avant à l'entrée du village de Voh; personne n'y habite. Tandis que cet agrandissement est resté spatialement très limité, la tribu s'est vue attribuer en 1989 un GIE (Groupement d'intérêt économique) ${ }^{7}$ d'une taille conséquente (1,610 ha): le GIE Pinjen (Figure 3). Cette attribution foncière fait suite à une histoire de spoliation et de « cantonnement » dans la région.

En 1877, une réserve nommée «Pinhadjen » formait une enclave dans une propriété privée mais n'était pas vraiment instituée comme « réserve », car les clans n'y étaient pas installés. En 1899, une réserve d'« Oundjo » est démarquée. Parallèlement, certains terrains sur Pinjen sont acquis par des colons français et par l'Etat français. La réserve tribale d'Oundjo est finalement créée en 1901 (Kowasch 2010: 330). Entre 1900 et 1920, les populations kanak sont chassées par le bétail des colons français qui détruit les champs. Les Kanak menacent de tuer le bétail, si leurs propriétaires n'installent pas d'enclos pour l'empêcher de divaguer, mais les éleveurs ne réagissent pas. Lors de l'insurrection de 1917, les Kanak tentent en vain de revendiquer la presqu'île de Pinjen: ils subissent la répression de l'administration coloniale. En 1929, l'ensemble des parcelles est acquis par la compagnie multinationale Ballande qui y installe une entreprise d'élevage de bétail (Horowitz 2003: 131). Durant la guerre civile de 1983 à 1988, les clans résidents à Oundjo revendiquent à nouveau les terres de la presqu'île. Puis, les membres de plusieurs clans d'Oundjo forment un GIE élevage afin de réclamer Pinjen. Ce GIE qui était un instrument de la revendication foncière prit le nom « GEO 6 », car six clans disaient avoir habité à Pinjen: les Diéla, les Tchaounyane, les Tidjite, les Poadataba, les Voudjo et les Gouneboadjane. Le 30 octobre 1989, le GIE «GEO 6 » devient définitivement propriétaire de la péninsule, une parcelle de 1,610 ha est rétrocédée. Tous les clans de la tribu d'Oundjo furent représentés dans le GIE à l'époque, à part les Dianaï qui n'ont pas de terres coutumières à Oundjo. Le GIE Pinjen est géré par un bureau et un comité d'administration composé d'un président, d'un vice-président, d'un gérant, d'un cogérant, d'un trésorier et d'un secrétaire, tous issus de la tribu d'Oundjo. Aux membres du bureau d'administration s'ajoutent les simples membres, une ou deux personnes par clan.

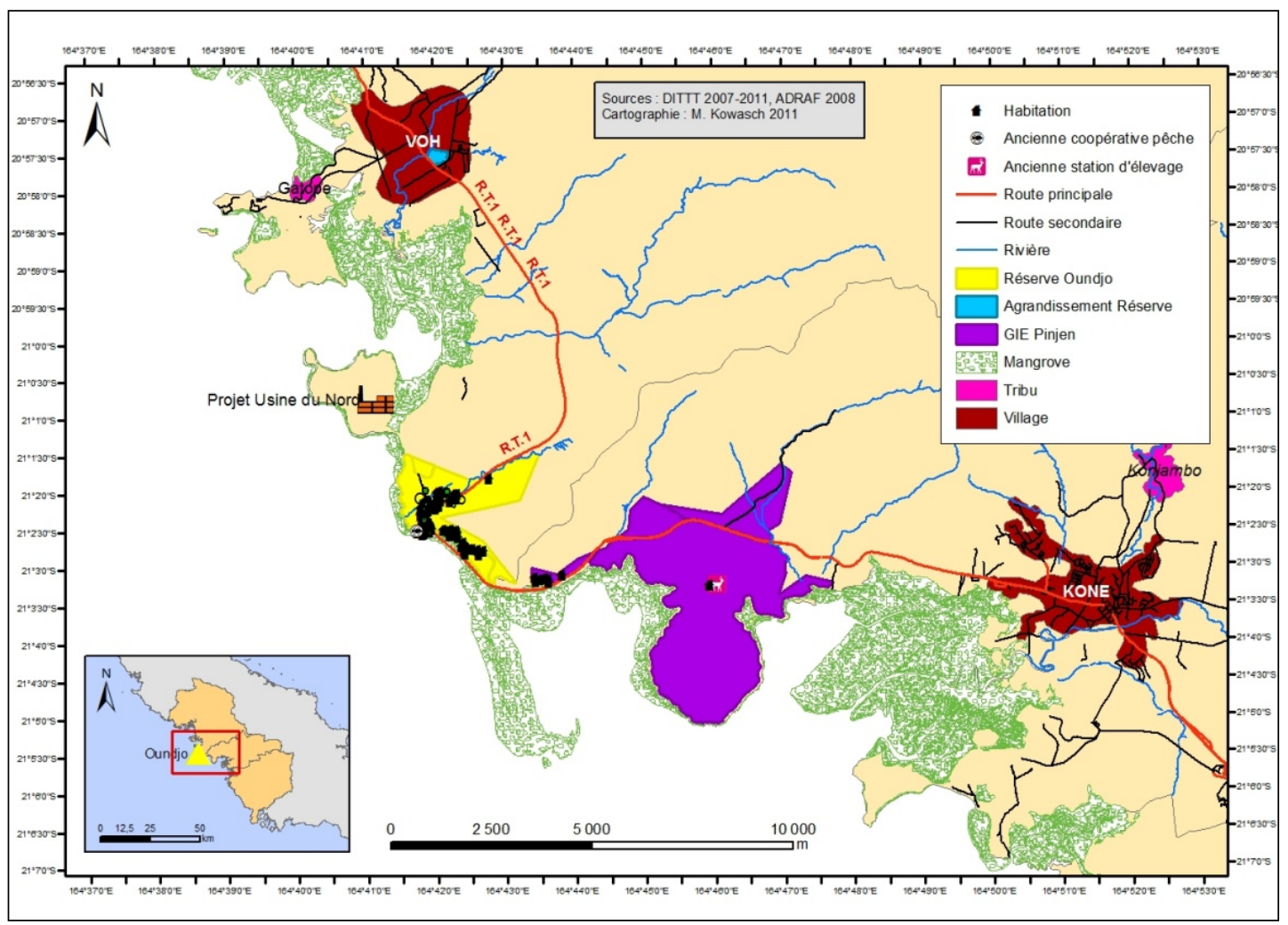

Figure 3 : Les terres coutumières de la tribu d'Oundjo. Source: M. Kowasch.

\footnotetext{
${ }^{7}$ Au début de la réforme foncière en Nouvelle-Calédonie, et avant la création des GDPL, les terres furent attribuées
} sous forme de GIE aux clans kanak. 
La presqu'île de Pinjen possède plusieurs avantages pour sa mise en valeur (Figure 3):

- sa situation géographique proche du village de Koné, le chef-lieu de la Province Nord,

- la Route Territoriale (RT) 1 passe juste derrière la presqu'île,

- elle est très peu vallonnée,

- le fait d'être une presquîle: elle est accessible par bateau.

L'intégration dans le développement socio-économique de la zone VKP (Voh-Koné-Pouembout, Figure 2) devrait alors se faire sans difficulté. La SMSP y a songé : En 1999, la société organise un meeting à Oundjo pour demander l'accord de la tribu pour construire la future « usine du Nord » à Pinjen. Selon L. Horowitz, les clans Tchaounyane, Diéla, Tidjite et Poadataba se tiennent d'abord en recul, mais acceptent finalement par un geste coutumier:

Members of the Caunyan, Diela, Tijit and Pwadaatraba clans stepped forward as the customary landowners of the peninsula. After some reflection, senior members of these clans gave their consent through a customary gesture, which Falconbridge accepted as evidence that the community had given them permission to conduct feasibility studies for the refinery construction (Horowitz 2003: 132).

Le clan Tidjite n'était plus représenté dans le GIE au moment de la demande de la part de la SMSP. Les clans Tchaounyane, Diéla et Poadataba ne pouvant pas décider seuls de l'avenir de la presqu'île, le bureau du GIE rappelle en mars 2010 qu'il est le propriétaire légal de la presqu'île, et certains membres font un blocage du site afin d'empêcher l'opérateur minier et ses sous-traitants d'entrer sur la propriété. Ensuite, plusieurs réunions eurent lieu au sein de la tribu où chaque parti demande que ses droits soient respectés. Le choix « Pinjen » de la SMSP divise le GIE. Au final, la majorité des membres du GIE se prononce contre le projet «usine du Nord" à Pinjen, tandis qu'une minorité est favorable à la construction. Plusieurs raisons expliquent leur décision (Kowasch 2010: 332). Premièrement, les terres de Pinjen avaient une vocation agricole à l'époque quand le GIE a récupéré la presqu'île. Deuxièmement, «On a pas voulu (l'usine) à Pinjen, parce que c'est la terre de nos ancêtres » (Communication personnelle à Oundjo le 4 janvier 2009). Troisièmement, la presqu'île était un " point chaud » de la revendication foncière lors de la guerre civile dans les années 1980; elle renvoie à l'histoire douloureuse de la spoliation et de la rétrocession des terres. Pinjen est donc un lieu « symbolique » pour cette lutte. Une fois la terre attribuée, les clans ne voulaient pas la céder à nouveau: ils ont " préféré laisser Pinjen aux clans » (Communication personnelle à Oundjo le 7 janvier 2009). Horowitz (2009: 6) évoque encore d'autres raisons comme par exemple la crainte que les ressources marines soient impactées. Les membres du GIE qui étaient contre "le choix Pinjen" savaient la majorité de la tribu en accord avec eux. Certains membres du GIE cependant se sont exprimés en la faveur de l'implantation de l'usine à Pinjen. L'argument principal visait les retombées économiques: « On aurait pu avoir beaucoup de retombées. Cela aurait facilité le travail à la tribu. » explique un ancien membre du GIE (Communication personnelle à Oundjo le 19 janvier 2009). Quant à la SMSP, elle tenta à plusieurs reprises de persuader les responsables du GIE de laisser ou de vendre les terres de Pinjen au groupe minier. Après que le GIE ait refusé l'offre de la SMSP de céder les terres de la presqu'île, le groupe minier s'oriente vers la presqu'île de Vavouto au nord d'Oundjo (Figure 3) où on achète les terres de droit commun à une famille Martin. La construction de la future usine du Nord put commencer, même si certaines revendications foncières de clans kanak existent également à Vavouto.

Ce désaccord sur l'avenir de la presquîile de Pinjen se montre aussi dans la cartographie des sites sacrés (Figures 4 et 5). Une multitude de légitimités foncières qui se superposent ne facilite pas un récit commun. Les deux cartes précédentes furent réalisées avec deux représentants de clans ayant ou réclamant une légitimité foncière sur la presqu'île. On aperçoit une différence concernant le nombre et aussi concernant l'emplacement des sites sacrés. La carte en haut fut réalisée avec quelqu'un favorable à l'implantation de l'usine du Nord sur Pinjen; la carte en bas avec quelqu'un opposé à la mise en place. Le nombre de sites sacrés sur la carte à droite est nettement plus élevée et démontre que la personne accorde une plus grande importance à la valeur patrimoniale - une importance que la personne ne veut pas qu'elle soit dépréciée.

Une reconnaissance foncière découle de la mise en place d'un projet économique comme l'usine du Nord, car les coutumiers acteurs du projet deviennent un interlocuteur privilégié des autorités publiques. A cette reconnaissance foncière s'ajoute le souhait de bénéficier sur le plan financier. C'est pour cela, des revendications foncières, en sommeil depuis des décennies, peuvent émerger lors un projet économique est annoncé. La rétrocession de terres depuis la réforme foncière entamée dans les années 1970 n'empêche alors pas que des revendications apparaissent même sur des terres attribuées depuis longtemps. 

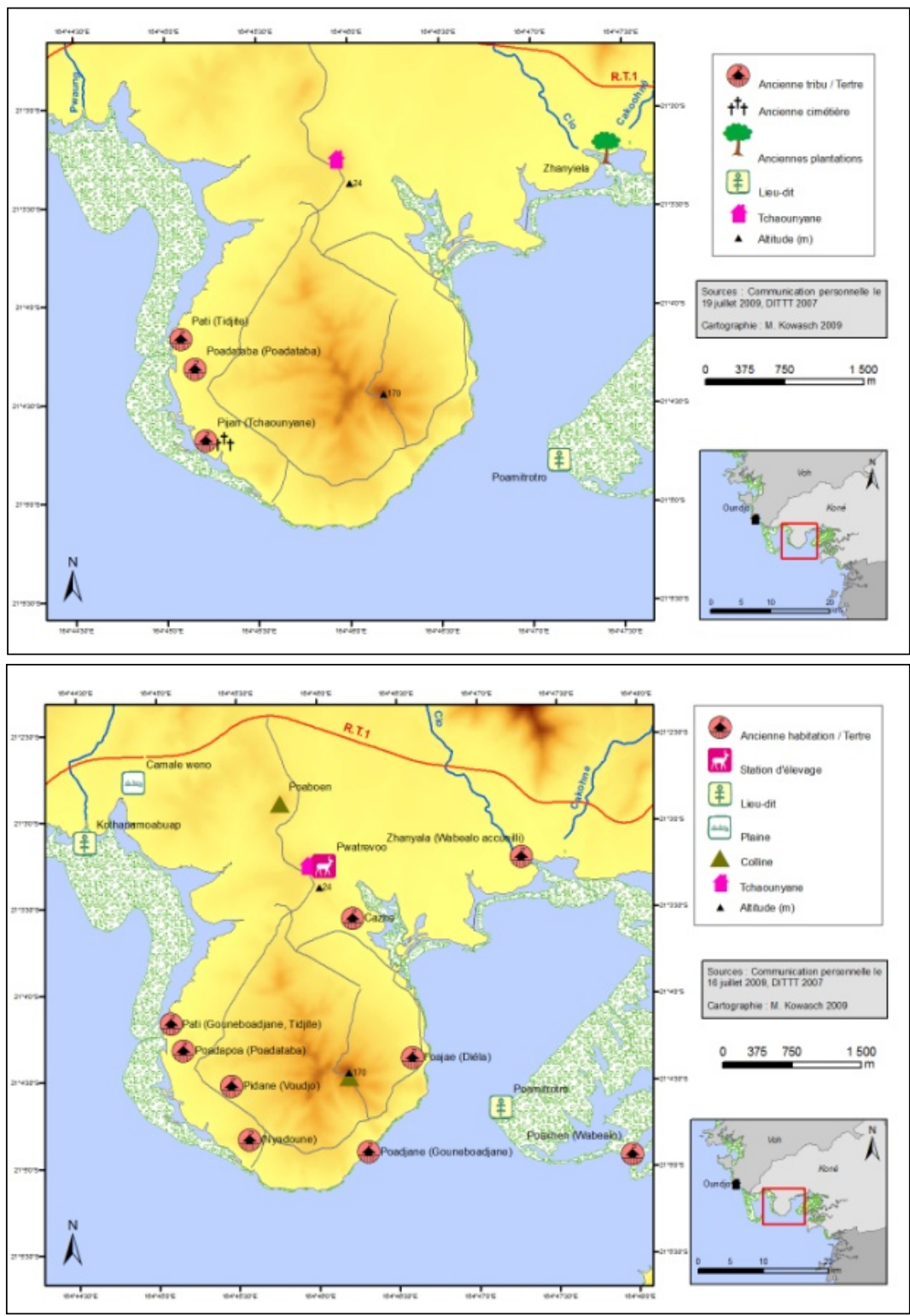

Figures 4 et 5 : Cartes des sites sacrés sur la presqu'île de Pinjen, réalisées par deux clans différents. Source: M. Kowasch.

\section{La tribu de Baco}

La tribu de Baco se situe à environ quatre kilomètres du village de Koné en province Nord. Cette proximité donne à Baco un caractère péri-urbain qui diffère d'autres tribus. Baco se situe à environ $15 \mathrm{~km}$ (9.3 miles) de la mer, à l'intérieur des terres, entre mer et montagne. Selon le bureau d'études « Emergences », le nombre d'habitants s'élève à 562 en décembre 2008 (Emergences 2009: 13). La tribu de Baco compte 22 clans regroupés en quatre "grands clans »: Wabealo, Baco, Poawidapthia et Poaxu. Les habitations de la tribu de Baco sont assez dispersées. La situation foncière de la tribu est particulièrement intéressante (Figure 6). Les terres coutumières couvrent une surface totale de 4,264 ha, dont 229 ha pour l'ancienne réserve, 1,987 ha pour les quatre GDPL claniques et 1,482 ha pour le GDPL tribal de Tiaoué-Pouembout. Il y a en effet quatre GDPL claniques: le GDPL Wabealo pour le clan Wabealo, le GDPL clanique de Bako pour le clan Bako, le GDPL Poavidapthia pour le clan Poavidapthia et le GDPL Poadjane pour le clan Poaxu. Et l'ADRAF compte attribuer deux autres GDPL aux clans de Baco (Figure 6), l'un tribal, l'autre clanique.

La tribu jouit, grâce à la taille conséquente de ses terres et par sa situation proche du chef-lieu de la province Nord, des meilleures conditions requises pour une intégration de ses GDPL dans le développement socio-économique de la zone VKP. La tribu de Baco s'engagea alors dans plusieurs projets de développement économique. Un exemple est la mise en valeur des terres du GDPL clanique de 
Bako (517 ha). Le plan d'aménagement pour une partie du GDPL comprend trois zones, sur une surface totale de 48 ha: habitat, activités et équipements. Entre autres seront construits une banque, une salle de spectacle, un cinéma de trois salles, une École d'Arts et un musée Lapita. A cela s'ajoute une zone commerciale, une gare routière à vocation provinciale et territoriale et une zone d'habitat (constituée dans un premier temps de 10 à 20 logements). Selon Patricia Goa, députée à la province Nord et membre du GDPL clanique de Bako, "la notion culturelle est aussi importante que la notion économique » (Communication lors d'une réunion du 26 septembre 2008). C'est pourquoi, elle souhaite que le musée Lapita, qui expose les origines du peuplement calédonien, soit une sorte de «vitrine » dans l'aménagement du GDPL.

La SAEML ${ }^{8}$ Grand Projet VKP fut mandatée de réaliser la gestion et l'administration du projet. Pour chacune des unités, un bail sera signé. Les terrains seront loués pour une période déterminée, sauf pour les projets « musée Lapita » et « École d'Arts ». Comme il s'agit de projets de représentation de la culture kanak, le clan Bako cessa les terres à titre gratuit pour que ces deux projets soient réalisés. En contrepartie, le clan demande que «tout ce qui regarde l'entretien intérieur et extérieur des futurs bâtiments, que le GDPL puisse être consulté » (Procès-verbal de la réunion du dimanche, 15 avril 2007, entre la SAEML GP VKP et le clan Bako). En outre, le clan exige que l'on prenne en compte les artisans locaux dans la mesure du possible dans la programmation des travaux et le bassin de l'emploi de la commune. La cession à titre gratuit s'explique également par le fait que la valeur patrimoniale du terrain ne fut pas modifiée. Aucune entreprise à but lucratif ne s'installe sur le terrain qui ne prendra donc pas de valeur d'usage économique.

La mise en valeur de terres coutumières s'inscrit dans l'objectif politique d'un rééquilibrage du territoire calédonien en faveur de la population kanak, mais elle poursuit parallèlement une logique rentière. "C'est notre coin de business ", réclame le mandataire d'un GDPL à la tribu de Baco (Communication personnelle, 2009). Le développement économique sur une terre coutumière sert à améliorer la condition de vie, de la même façon qu'une personne fait pousser des tubercules dans son champ pour le bien de la famille et du clan. Winslow expliqua déjà en 1995 que les dirigeants kanak étaient « opposés à l'idée du 'profit individuel' » (Winslow 1995: 9).
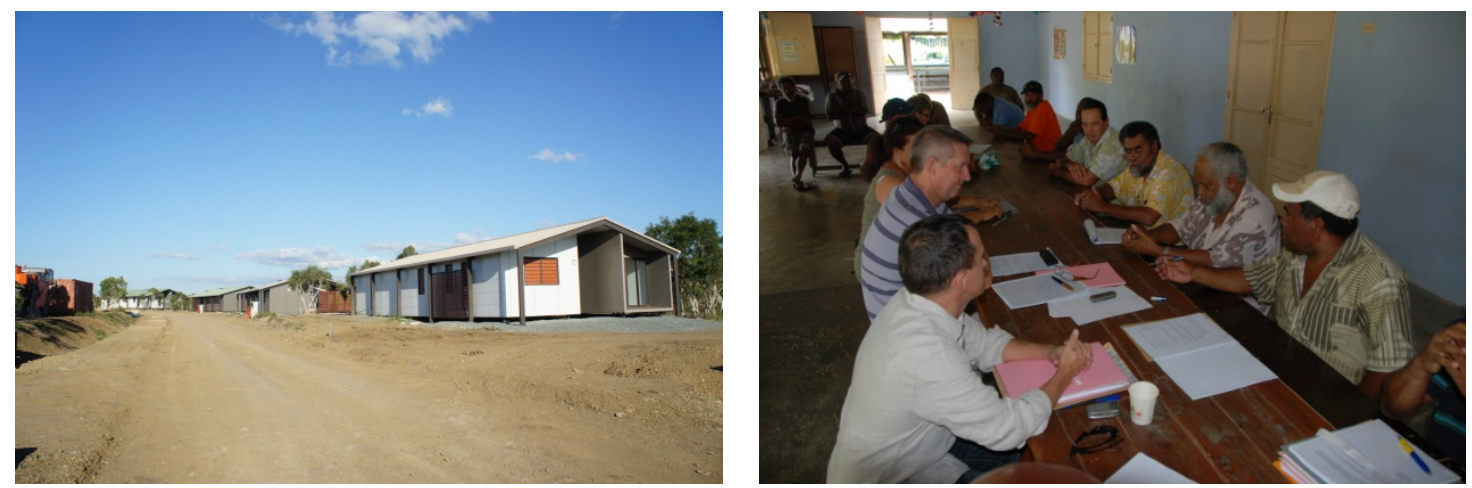

Images 2 et 3 : Construction d'un lotissement locatif sur le GDPL clanique de Bako (gauche), Réunion à la maison commune de la tribu Baco entre la SAEML GP VKP, les maires de la zone VKP et les coutumiers de Baco (droite). Sources: M. Kowasch 2011 (photo à gauche) et 2008 (photo à droite).

Ce profit individuel est considéré comme un «schéma de référence » venu de la France qui reste aux yeux de beaucoup de Kanak un «État colonial ». En mettant en valeur une partie du GDPL clanique de Bako, le bureau d'administration du GDPL tente à faire profiter le clan dans son ensemble. Dans ce sens, il convient de parler d'une "participation communautaire " au projet Koniambo qui comprend le développement de la zone Voh-Koné-Pouembout (VKP) (Images 2,3). Cette "participation communautaire ", qui convient mieux aux valeurs traditionnelles de la société kanak qu'une «participation individuelle » (par exemple en créant une entreprise privée), nécessite par contre le consensus du groupe de référence lignager ou clanique. En cas de désaccord, le projet n'avancera pas, il restera en «stand-by ». A Pinjen, le projet d'implantation de la future usine du Nord fut complètement abandonné, car le groupe minier n'avait pas le temps d'attendre; il choisit un autre site industriel. L'aménagement d'autres GDPL est bloqué, car les clans sont en conflit.

La démarche pour l'aménagement du GDPL clanique de Bako démontre que l'initiative de la mise en valeur du foncier coutumier vient généralement des autorités publiques de la province Nord et non du clan. La SAEML VKP est maître d'ouvrage délégué pour le projet de l'aménagement du GDPL clanique de Bako. Les clans membres du GDPL délèguent ainsi la mise en œuvre du projet à la puissance communale ou provinciale. Il s'agit donc plutôt d'une « participation dirigée » des clans concernés, car le maître d'ouvrage délégué prend les décisions pour le programme d'aménagement, y compris

\footnotetext{
${ }^{8}$ SAEML = Société anonyme d'économie mixte locale
} 
l'emplacement des équipements. Donc, les coutumiers n'agissent pas en "investisseurs », mais en « rentiers ». La logique économique kanak n'est pas celle de l'investissement à long terme, mais plutôt de la rentabilité immédiate, elle n'est pas « productiviste », mais « rentière » (Kowasch 2010: 464).

« Le coin de business » se trouvant toujours à l'extérieur de l'espace de vie quotidienne, il existe une certaine appréhension à voir arriver des nouveaux arrivants. A cela s'ajoute le désir de ne pas se mélanger à eux, on appelle ce phénomène " le syndrome îlien ». D'après cette idée, la tribu devrait rester comme elle est, le développement économique et l'accueil de nouvelles populations dans des lotissements locatifs se fait autour. La tribu kanak ressemble de ce fait à une « île » entourée de collectivités et de terrains de droit commun où se réalise pour l'essentiel l'activité économique. Une application du modèle d'économie capitaliste où le profit individuel prime à l'intérieur de la tribu semble actuellement impossible. Si elle se fait, elle ne pourra être décidée que par ses résidents, donc par les autorités coutumières de la tribu. Parallèlement, il faut souligner le manque d'espace pour réaliser des projets de développement en tribu. Les réserves, fruits amers des spoliations foncières coloniales de la fin du $19^{\mathrm{e}}$ début du $20^{\mathrm{e}}$ siècle, représentent un espace très restreint. De plus, les terres agricoles ne sont souvent pas d'une bonne qualité. L'aménagement des terres coutumières, comme par exemple celui du GDPL clanique de Bako, s'inscrit alors dans une stratégie de déconcentration du peuplement, une " reconquête " de l'espace qui entre dans la logique actuelle de décolonisation négociée. Les projets de développement en dehors de l'espace « intime » des tribus constituent une manière de réaliser cette reconquête et réappropriation de l'espace (Figure 6, Images 2,3) (Kowasch 2012: 16).

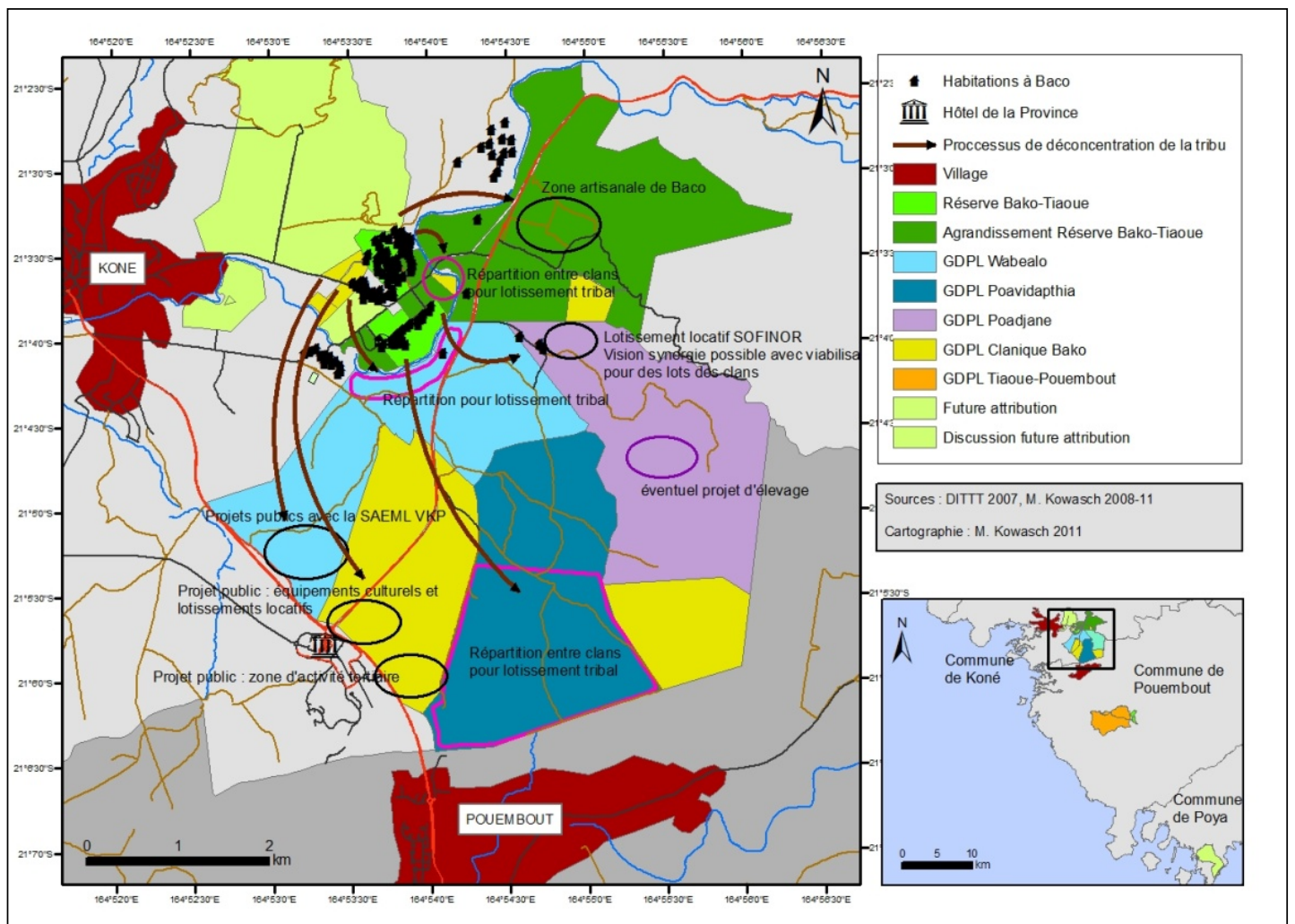

Figure 6 : Mise en valeur des terres coutumières de la tribu de Baco. Source: M. Kowasch.

\section{La fédération coutumière « Djelawe»}

« Djelawe », ce qui signifie « bord de mer » ou « bord de l'eau » dans la langue « haveke » d'Oundjo et Gatope ${ }^{9}$, fut créée en 2006. La "Fédération coutumière Djelawe », son nom officiel, se présente comme une association pour la préservation de la nature, mais elle poursuit principalement l'objectif d'une reconnaissance foncière de ses clans membres. D'après le président de «Djelawe », la fédération fait suite à une réunion avec l'ADRAF et la province Nord en 2003 où on aurait conseillé à son clan de mettre en place une représentation pour les gens du bord de mer (Communication personnelle le 13 juillet 2007). Les membres de la fédération sont les GDPL et clans suivants ${ }^{10}$ (Figure 7): le GDPL Cathete Ma

9 La tribu de Gatope se situe au nord d'Oundjo et de la presqu'île de Vavouto, à quelques kilomètres seulement du village de Voh en bord de mer.

${ }^{10}$ Communication personnelle du président de « Djelwae » le 13 juillet 2007), ADRAF juillet 2009. 
Poahmeane $^{11}$, le GDPL Xaapeta, le « clan Pinjen » ${ }^{12}$, le GDPL Taa-Ma-Pwanefuk, le GDPL XuutiXuthapet, le clan Tanecout (Témala-Ouélisse), le GDPL Kamacouth et le GDPL Kanecout. Seuls le GDPL Xuuti-Xuthapet et le GDPL Kanecout sont des GDPL attributaires de terres. La revendication du GDPL Xaatepa fut enregistrée par l'ADRAF, mais les discussions perdurent en 2012. Les autres revendications représentent plutôt des zones d'influences des clans membres du GDPL respectif. Pour le GDPL Xuuti-Xuthapet par exemple, une attribution foncière eut déjà lieu, mais cette rétrocession ne correspond pas à la zone d'influence marquée dans la carte suivante. La presqu'île de Pinjen fut également déjà attribuée sous forme de GIE à un groupement de clans résidant à la tribu d'Oundjo. Cependant, un certain clan d'Oundjo continue à réclamer une légitimité foncière; le président de " Djelawe » l'appelle "clan Pinjen " ${ }^{13}$. Enfin, on constate que quasiment l'ensemble du littoral de la zone Voh-KonéPouembout (VKP) est revendiqué par les clans membres de «Djelawe », ce que démontre la carte cidessous (Figure 7). Il existe par contre des clans vivant en bord de mer qui ne sont pas membres de « Djelawe »; eux aussi réclament une légitimité foncière, un « droit à l'endroit ». Certains clans formèrent déjà un GDPL afin d'exprimer leur revendication auprès de l'ADRAF, par exemple le « GDPL Vavouto ».

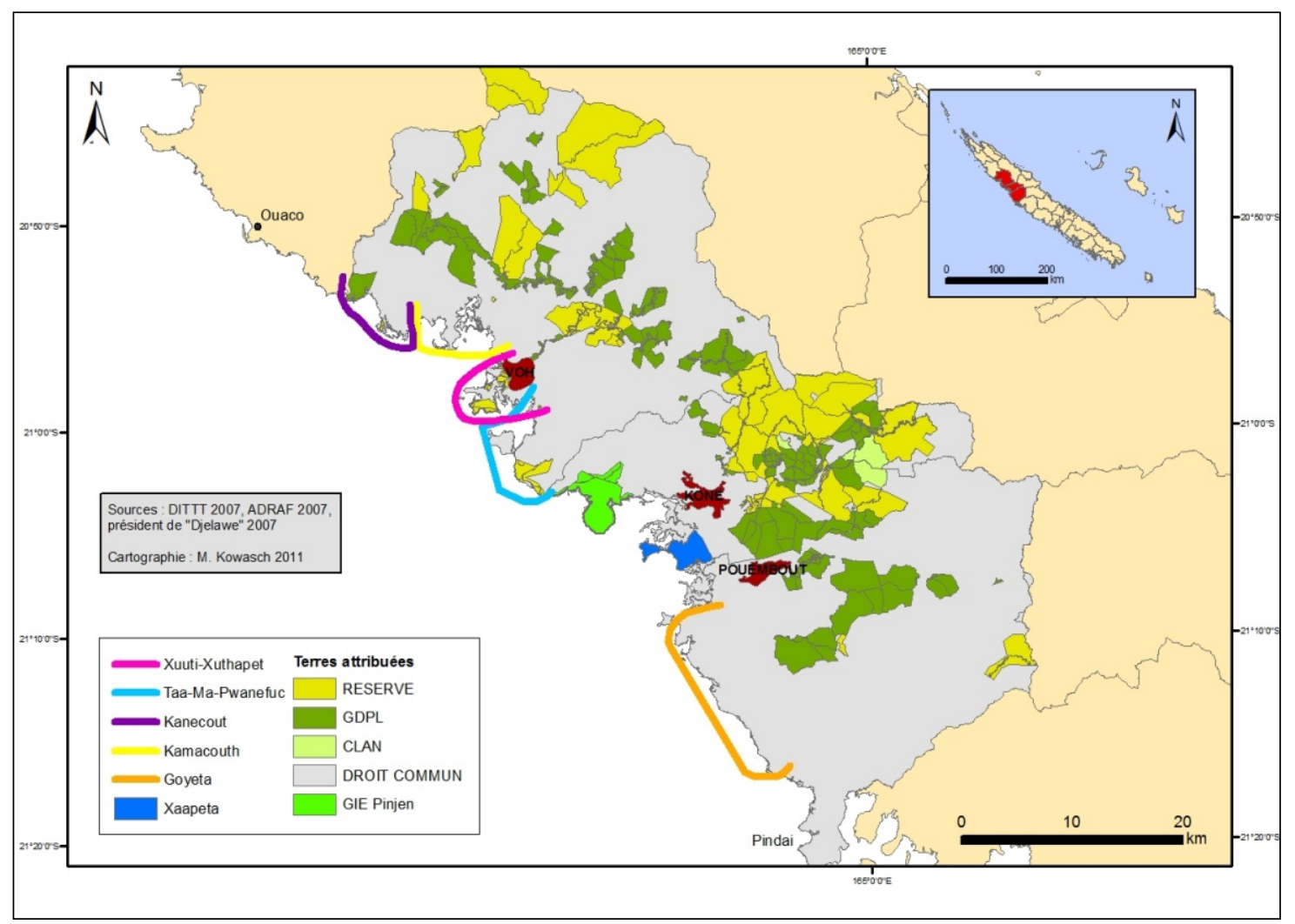

Figure 7 : Les revendications foncières de la fédération « Djelawe » dans la zone VKP. Source: M. Kowasch.

L'association « Djelawe » est un invité régulier du Comité Environnement Koniambo (CEK) dont l'objectif est de traiter tout sujet ayant trait aux questions environnementales liées au projet « usine du Nord ». De plus, le comité qui est une structure appartenant au groupe minier KNS devrait contribuer au proccessus de concertation et d'information auprès de la population locale. Selon un membre de " Djelawe », la fédération souhaite être chargée de la surveillance du lagon (Communication personnelle le 19 janvier 2009). « Djelawe » posa une demande officielle à l'opérateur minier KNS pour qu'elle puisse mettre en place un système de surveillance avec deux bateaux qui pourront surveiller une zone très large, entre Pindaï au Sud et Ouaco au Nord (Figure 7). Tous les bateaux qui viennent de l'extérieur, devraient payer un droit de passage. Ce sont les clans reconnus sur « leurs » terres qui devraient, selon le membre, contrôler les accès à la mer (wharfs, rampes de mise à l'eau, etc.). L'espace coutumier s'étend donc naturellement de l'espace terrestre à l'espace maritime. L'idée que les clans reconnus sur « leurs » terres

\footnotetext{
${ }^{11}$ Dans l'interview, le président parlait du «GDPL Goyeta ", mais le clan Téin d'Oundjo qui est membre de " Djelawe » fonda entre temps son propre GDPL: "Cathete Ma Poahmeane ». cependant, le " GDPL Goyeta » continue à exister, des clans d'autres tribus y sont membres (Information de l'ADRAF, juillet 2009).

${ }^{12}$ Le « clan Pinjen » désigne le clan Tchaounyane résidant à la tribu d'Oundjo.

${ }^{13}$ Une famille de ce clan habite dans l'ancienne station d'élevage sur la presqu'île (Figure 5).
} 
rejoint le rôle que le président du « Djelawe » accorde à son association: « accompagner les gens du bord de mer dans leurs projets pour faire reconnaître leurs droits " (Communication personnelle le 13 juillet 2007). La gestion des ressources marines et la protection de l'environnement ne représentent donc pas les premières préoccupations de l'association. L'objectif premier est plutôt la reconnaissance en tant qu'interlocuteur privilégié de l'industriel (ce qui se traduit par la participation aux réunions du CEK) ainsi que la reconnaissance de la légitimité foncière pour les zones revendiquées (ceci découle du fait d'être interlocuteur privilégié) et de bénéficier du projet Koniambo sur le plan financier. On pourrait ainsi comprendre « Djelawe » comme une sorte d'alliance destinée à se positionner dans le jeu d'influence que représentent les revendications foncières des populations kanak dans la zone VKP. Le discours environnementaliste est un outil pour atteindre l'objectif d'une reconnaissance sur le plan foncier.

Généralement, le contrôle d'un espace marin est la tâche des autorités publiques. Si on chargeait les clans membres de «Djelawe » de surveiller le lagon entre Ouaco et Pindaï, ils seraient donc reconnus en tant que «propriétaires terriens » de l'espace marin. Reste le problème que tous les clans vivant en bord de mer ne sont pas membres de «Djelawe », entre autres des clans d'Oundjo et Gatope. En 2010, le groupe minier KNS charge la SAS Sowemar (détenue à 48,5\% par la SOFINOR, à 46,5\% par le clan Poithily de Gatope et à 5\% par la SCP Bord de Mer) d'assurer la sécurité autour du port de Vavouto. La surveillance de la zone autour du port privé de Vavouto sera alors réalisé par une société privée, détenu en partie par un clan membre de «Djelawe». Et la SAS Sowemar permet au clan Poithily d'être un interlocuteur privilégié de l'industriel.

En 2009, la fédération crée même une petite société de sous-traitance «Djelawe Maintenance ». Cette petite entreprise est chargée de la maintenance d'engins sur le chantier de Vavouto et la mine du Koniambo. « Djelawe » participe ainsi activement au projet Koniambo, touche des bénéfices et représente un interlocuteur pour l'opérateur minier.

\section{Co-existance de sites sacrés et d'activités minières et instrumentalisation du discours environnementaliste}

L'existence de sites sacrés sur les terres où fut découvertes des ressources minières fut déjà décrite par K. Doohan (2008) dans son livre «Making things come good» après avoir travaillé dans le Kimberley (nord-ouest de l'Australie):

The site where the diamond-bearing lamproite pipe occurs is called (...), in English, Barramundi Gap. Barramundi Gap is of significance to the local Aboriginal people, and especially women, because it is one of the numerous resting places of the female Barramundi creative Dreaming being. (...) Devil Devil Spring, a site of special significance to Aboriginal men, (...) there are secret-sacred aspects to this site (...).

Nous avons vu auparavant (étude des cas sur Oundjo/Pinjen) que l'existence de sites sacrés, d'anciennes habitations d'un clan par exemple, peut retarder, voire complètement faire annuler un projet économique. Comme une légitimité foncière en découle, l'implantation d'un projet sur les terres où on trouve les sites sacrés génère ou réveille bien souvent des conflits. Une légitimité foncière entraîne plus de prestige et éventuellement des royalties. La question déterminante est de connaître les facteurs qui font qu'un projet soit arrêté ou annulé à cause ou grâce à l'existence de sites sacrés. Plusieurs aspects rentrent en jeu: la détermination des acteurs, des clans "propriétaires terriens », mais aussi de l'industriel et des autorités politiques. Dans la plupart des cas, le projet continue. Malgré l'existence du «Western Australian Aboriginal Heritage Act 1972 » et l'existence de sites sacrés dans la vallée Fitzroy, le groupe AMAX continua par exemple ses activités d'extraction de pétrole à Noonkanbah, dans la région de Kimberley dans le nord-ouest de l'Australie, décrit par K. Doohan (2008) et Hawke et Gallagher (1989). Doohan nota en 2008 (p. 18-19):

The Noonkanbah dispute had begun when local Aborigines opposed the drilling programme proposed by the oil exploration significance on Noonkanbah Station. (...) Pea Hill was drilled, but no oil was found. (...) However, the State had effectively demonstrated both its will and its capacity to support resource development activities against Aboriginal people's efforts to protect their sacred sites and cultural heritage.

Dans le Sud de la Nouvelle-Calédonie, le projet d'exploitation minière et de la mise en place d'une usine de traitement (projet « Goro Nickel ») fut finalement acceptée, malgré l'existence de sites sacrés:

Dans ce site de la mine, il y a des sites tabous, des sites sacrés, des lieux de passage, des sentiers, des crânes, des coquillages, des toutoutes. C'est là que vivent les ancêtres. Il n'y a pas de langages pour lever les sites tabous. Nous, on ne pourra jamais donner notre accord pour que le projet aille sur ces sites. Si Goro Nickel le fait, il prend ses responsabilités, mais nous, on dit non. 
...exprima le grand chef de la tribu de Goro en juillet 2003 (http://www.rheebunuu.com). A la fin du combat entre les autorités coutumières et le groupe Vale Inco, les coutumiers signèrent un « pacte pour un développement durable du Grand Sud » qui prévoit la création d'une fondation d'entreprise, d'un observatoire de l'environnement et une pépinière. Le groupe Vale Inco versera environ 1 million d'euros par an à une fondation d'entreprises locales et s'engage au total avec environ 80 millions d'euros sur 30 ans dans différentes mesures de protection de l'environnement (formation de 8 techniciens environnementaux, programme de reboisement des zones périphériques à l'activité minière, collecte de graines, création de pépinières satellites, etc.).

Selon Donna Winslow, l'environnement naturel représente un instrument pour atteindre un but politique - la souveraineté: « Pour le Front d'Indépendance Kanak, l'utilisation de l'environnement naturel est désormais perçue comme une manière d'aboutir à un but spécifique - la souveraineté. » (Winslow 1995: 2) L'exploitation des ressources minières est donc un outil pour les leaders kanak du FLNKS d'avoir une base économique afin de lancer un processus d'émancipation politique. Le but de la mise en place d'une usine métallurgique n'est donc pas d'obtenir le plus de profit pour la compagnie minière, mais de développer l'ensemble du pays. Paul Néaoutyine, le président de la province Nord, disait ainsi dans son livre « L'indépendance au présent »:

Si nous devons entrer dans l'activité nickel, notre philosophie est que le minerai devrait être transformé ici. Si nous y parvenons, nous prouverons, un, que nous sommes capables de gérer un domaine d'activité essentiel pour le pays, deux, que le rééquilibrage ne peut se faire uniquement à coup de contrats de développement et d'aides de la France, sans parvenir à créer suffisamment de valeur ajoutée ici pour relayer le financement du développement (Néaoutyine 2006: 158).

En mettant en valeur leurs terres coutumières, les populations kanak participent activement au développement de la zone VKP. L'environnement (soit les terres mises en valeur) représente donc un outil pour l'intégration dans le développement de la zone et du pays dans son ensemble ainsi qu'un instrument pour faire avancer le processus d'émancipation politique. La dépendance financière de la France métropole pourra - grâce aux bénéfices du secteur nickel - diminuer, car les autorités publiques sont directement impliquées en détenant $51 \%$ des parts dans le projet Koniambo.

En utilisant l'environnement pour participer au développement économique du pays, les Kanak transforment la valeur de la terre. Ce remodelage du territoire entraîne souvent des conflits fonciers. On le voit dans chaque mise en valeur d'une terre coutumière. Une ascension sociale ne peut être réalisée de manière consensuelle qu'à travers la modification du territoire (Herrenschmidt 2003: 322). Mais la progression déconnectée du territoire, par exemple une création d'entreprise de gardiennage en soustraitance dans le système " occidentalisé », ne mènera pas à un changement d'identité et à une ascension au sein du groupe ou du clan. C'est donc la reconnaissance d'un clan à un certain endroit qui pourrait changer la perception territoriale et entraîner ce remodelage du territoire. Une évolution de l'identité du groupe en découlera. Les revendications foncières s'inscrivent dans un jeu d'influence qui a pour objectif de modifier le territoire au gain de son propre groupe. Le développement économique est instrumentalisé dans cet enjeu afin de modifier la perception territoriale. La création de l'association coutumière « Djelawe » en est un exemple.

\section{Revendication «autochtoniste» et transformation de la valeur de l'environnement}

Les revendications foncières de la fédération « Djelawe » démontrent la constante réactualisation de la notion de «propriété ». Les revendications foncières se basent sur l'ancienneté, c'est-à-dire sur le fait d'être le " premier occupant » de la terre. Les Kanak ainsi que d'autres populations autochtones réclament un contrôle du foncier où le clan est passé durant son histoire, mais également une propriété intellectuelle en ce qui concerne le patrimoine immatériel (chants, mythes, savoir-faire de sculpture, tressage, etc.). N. Castree décrit ces revendications dont on entend davantage parler depuis la Déclaration des Nations Unies sur les droits des peuples autochtones en $2007^{14}$ :

To simplify, many indigenous peoples are currently seeking to reappropriate three things that, historically, have been taken away from them: namely, parcels of land and water, material artefacts (e.g. ceremonial goods like masks), and knowledges (e.g. designs and medicinal remedies). Together, we can call these things physical, cultural and informational resources, ones that are highly valued by indigenous communities in a number of ways (instrumental, symbolic, moral and aesthetic). (Castree 2004: 151)

Castree discute ensuite la thèse si ces revendications foncières des peuples autochtones sont « régressives » ou « progressives ». Premièrement, il faut constater que les revendications réclament une sorte d' « exclusivité » sur la terre en question. D'autres populations sont alors exclues ce qui démontre

${ }^{14}$ http://www.un.org/esa/socdev/unpfii/_documents/faq_drips_fr.pdf 
d'ailleurs l'exemple de « Djelawe » dans le nord de la Nouvelle-Calédonie. M. Watts (1999, 2001), D. Massey (1999) et D. Harvey (2000) voient dans ces mouvements autochtonistes une tendance " regressive ", car la " bonne cause » (rétrocession de terres après une histoire triste de colonisation et de spoliation foncière) ne justifie pas une exclusion d'autres populations. Pour Castree (2004: 142, voir aussi Watts 1999), personne ne peut réclamer une exclusivité foncière, car tout identité est construite en rapport avec l'extérieur:

Relatedly, wish images and the identities they express need a location to call their own. Just as wish images may involve fantastical visions of a group's past and possible future, so the territorialisation of identities is equally fictive: claims to parcels of space can be legitimated with reference to spurious histories of 'homelands', 'birthrights', 'origins' and the like.

Le discours d'origine se trouve aussi chez les Kanak en Nouvelle-Calédonie, pour qui il est extrêmement important " d'où une personne vient ». Cela représente une notion d'identité et d'exclusivité. D. Massey (1999: 40) soutient l'argumentation de Watts: «I absolutely reject ... the claims to local exclusivity and the terms in which they ... are made. » Massey imagine l'espace, l'environnement « as the sphere of juxtaposition, or co-existence, of distinct narratives, as the product of power-filled social relations ... This is place as open, porous, hybrid ... ». Les deux auteurs, Massey et Watts voient à travers l'opposition entre « insiders » et « outsiders » un danger réel dans le programme politique du mouvement. $\mathrm{N}$. Castree réplique les arguments de Watts et Massey par le fait que la revendication foncière représente un langage que les autorités publiques - allochtones dans ce cas - comprennent. Deuxièmement, Castree (2004: 160) évoque le besoin de sécurité foncière, culturelle et matérielle qui peut être atteinte par un " contrôle foncier »: " Property rights are among the most legally secure and materially effective rights that individuals and groups can possess. These rights promise to be an important tool for those indigenous groups seeking to exert strong forms of place control. » Les peuples autochtones ont le droit de se distinguer des autres populations et de récupérer des ressources à valeur économique, des ressources que d'autres populations ont exploité pendant des siècles. Grâce à la rétrocession de terres, les populations autochtones peuvent donc acquérir une certaine sécurité matérielle. Cette sécurité pourra ensuite contribuer au maintien d'un certain mode de vie qui est menacé.

Pour le cas de la Nouvelle-Calédonie, les objectifs de la fédération «Djelawe » démontrent que les revendications foncières s'inscrivent dans un enjeu de perpétuelle réactualisation, dans un jeu d'influence avec un prestige qui est « régulièrement » renégociée. La notion d'une renégociation permanente renvoie à un constat de P.-Y. Le Meur (2010a: 109): « Elles (les lieux toponymiques) ne peuvent pas fonctionner sur la base d'un référent historique unique dans la mesure où elles disent, sur la base de légitimités ancrées dans le passé, des rapports de force actuels qui ne sont pas figés, mais sujets à des renégociations permanentes. En même temps, tout n'est pas négociable (...) ». Les enjeux sont donc plus complexe qu'une simple opposition entre «insiders » et " outsiders ", même si elle existe dans de nombreuses revendications et crée ainsi des conflits inter-claniques. Cette exclusivité foncière entraîne des conflits entre clans kanak et néglige la juxtaposition de légitimités foncières. L'exemple de Pinjen montre que chaque clan s'est construit une propre identité basée sur l'itinéraire et se fait donc une propre image de l'espace. Cette analyse rejoint la thèse de $\mathrm{N}$. Castree qui nota (2001: 12): «We have to live with the fact that different individuals and groups use different discourses to make sense of the same nature/s. These discourses do not reveal or hide the truths of nature but, rather, create their own truths. " Dans le cas de la presqu'île de Pinjen, chaque clan a sa propre "vérité historique » (Figures 4 et 5), ainsi cet exemple confirme la thèse de Castree. Ces différentes vérités et conflits autour de l'antériorité foncière nous amènent de nouveau à la construction et reconstruction de l'identité liée au remodelage du territoire qui peut être atteinte par une attribution foncière. Ce remodelage se traduit par une transformation de la valeur de l'environnement.

\section{Conclusion}

L'identité kanak est le fruit d'un long mûrissement de la territorialité. Elle est soumise à une construction et reconstruction perpétuelle. L'histoire de l'itinéraire clanique représente le fondement de cette identité qui n'est pas niée en participant au développement économique. La réponse à l'assimilation des profondes transformations sociales et culturelles qu'apporte le développement de l'industrie du nickel en Nouvelle-Calédonie pourrait être la suivante: changer en restant soi-même. Un entrepreneur de la tribu d'Oundjo exprime ses angoisses envers les transformations: " Ça va nous faire peur. On n'a pas l'habitude de ce mode de vie ... Ça va bouleverser notre mode de vie, mais on est obligé de faire » (Communication personnelle le 6 janvier 2009). Dans ce sens, le syndrome îlien aide à préserver les valeurs du passé et les sites culturels à l'intérieur de l'espace « intime » tout en participant au développement économique au lieu de s'évanouir dans le monde capitaliste.

L'existence de sites sacrés sur les terres où doit se réaliser un projet économique au sens large, entraîne par contre souvent des conflits, car une légitimité foncière (un " droit à l'endroit ») découle de ces sites. Et une reconnaissance foncière signifie prestige et éventuellement des royalties. Une ascension sociale au sein de la société kanak n'est possible que par un remodelage du territoire. Ainsi, la mise en valeur d'une terre coutumière représente un moyen de "monter l'échelle sociale » quand on devient « interlocuteur privilégié » de l'opérateur industriel ou des autorités publics chargés du projet. De l'autre 
côté, le remodelage du territoire peut occasionner une évacuation de la valeur patrimoniale, si les sites culturels ne sont pas reconnus et enlevés ou détruits. Le clan ayant une légitimité foncière renonce donc au modèle mélanésien «traditionnel » en faveur du modèle de transition, voire du modèle économique occidental classique (Figure 1). Les deux exemples étudiées, Oundjo et Baco, démontrent que la valeur de l'environnement n'est pas la même pour les clans concernés; tandis que les lieux sacrés sont très importants pour les uns, ils n'ont aucune valeur pour d'autres. Le seuil quand un lieu sacré est abandonné pour des bénéfices financiers est fluctuant et dépend de l'histoire du clan ainsi que de l'actuel contexte culturel et socio-économique. Il convient de poser la question si certains lieux sacrés ne devraient pas être protégés par les institutions publiques avant qu'ils soient définitivement perdus, car ces sites possèdent une valeur collective. G. Bridge (2004: 229) se penche sur cette question de protection: « (...) an emerging consensus that some ecologically and culturally protected areas should be off-limits to mining and that mineral development is not an unalloyed benefit in all circumstances, (...) ». Mais G. Bridge se demande comment et qui pourra déterminer ce genre « no-go areas ».

Les conflits qui découlent des revendications émergent, car elles se basent sur une certaine exclusivité du peuple premier ou dans l'exemple de Pinjen ou de « Djelawe » de certains clans ayant vécu à l'endroit. Cette exclusivité exclut par définition d'autres clans. En même temps, il faut constater que la rétrocession foncière s'explique par une histoire coloniale douloureuse avec des spoliations foncières et des exploitations, voire éradication de ressources naturelles sans l'accord des populations vivant sur place. De plus, les revendications ne réclament pas forcément une exclusivité pour un clan, car plusieurs clans se regroupent afin de demander une rétrocession collective. De plus, l'attribution représente une certaine sécurité matérielle pour les nouveaux "propriétaires » (espace pour des nouvelles habitations par exemple).

Dans la majorité des cas, les revendications foncières des clans kanak ne sont pas le résultat d'un besoin économique (Doumenge 2003). La récupération de terres n'entraîne donc pas automatiquement la mise en valeur de ces terres; elle répond plutôt à une reconnaissance de l'histoire clanique, donc à une légitimité foncière. Les objectifs de la fédération coutumière « Djelawe » le démontrent. Depuis quelques temps, les autorités coutumières utilisent de plus en plus souvent un discours environnementaliste. La protection de l'environnement est devenue une véritable préoccupation, car le pays enregistre la mise en place de deux nouvelles usine de traitement de nickel et à terme un triplement de sa production métallurgique. Ce discours environnementaliste est cependant aussi un instrument pour atteindre une rente (minière), c'est-à-dire des royalties ainsi qu'une certaine légitimité dont découle un plus de prestige. P.-Y. Le Meur pense qu'il existe même une stratégie d'apposer son label de protection de l'environnement « pour s'en approprier les bénéfices symboliques » (Le Meur 2010b: 96). L'environnement devient donc un outil pour avoir des royalties. Vice versa, l'industriel pourrait polluer pour exclure le site du domaine protégé. Cette instrumentalisation, expliquée à l'exemple de « Djelawe », rejoint l'analyse de N. Castree dans son ouvrage «Social Nature » (2001: 9): « (...) it's often the case that claims about nature - and actions based upon those claims - can serve as instruments of power and domination ». Ce jeu d'influence autour du pouvoir et du prestige se traduit dans le conflit concernant la mise en place de la future usine du Nord sur la presqu'île de Pinjen; ce projet fut instrumentalisé pour des fins « politico-foncières ». Mais on pourrait également interpréter que c'est le projet métallurgique qui fit resurgir le conflit foncier.

Ainsi, les revendications foncières, les conflits, les négociations et le remodelage du territoire résultant du développement de l'industrie du nickel en Nouvelle-Calédonie représentent un exemple pour d'autres territoires où des populations autochtones sont confrontées à une exploitation de "leurs » ressources naturelles. Enfin, on peut conclure que tous les enjeux autour de la revendication foncière et de la transformation de la valeur environnementale démontrent que l'aspect socio-économique de l'environnement prime sur l'aspect bio-physique.

\section{Bibliographie}

ADRAF. 2000. La réforme foncière en Nouvelle-Calédonie 1978-1998. Nouméa: Éditions Grains de Sable.

Bonnemaison, J.; établi par Maud Lasseur et Christel Thibault. 2000. La géographie culturelle, Cours de l'université Paris IV - Sorbonne 1994-1997. Paris: Editions du Comité des travaux historiques et scientifiques (C.T.H.S.).

Bridge, G. 2004. Contested terrain: mining and the environment. Annual Review of Environment and Resources 29: 205-259.

Carnuccini, A.-S. et D. Guillaud. 1999. Les fumées de l'usine - un projet métallurgique à l'appui du rééquilibrage. In David, G., Guillaud, D. et P. Pillon (eds.) La Nouvelle-Calédonie à la croisée des chemins: 1989 - 1997. Nouméa: Société des Océanistes/IRD. Pp 83-99.

Castells, M. 1997. The power of identity. Oxford: Blackwell.

Castree, N. 2001. Socializing nature: theory, practice, and politics. In Castree, N. and B. Braun (eds.) Social nature: theory, practice, and politics. Oxford: Blackwell. Pp 1-21.

Castree, N. 2004. Differential geographies: place, indigenous rights and 'local' resources. Political Geography 23: 133-167. 
Constanza, R., D'Arge, R., De Groot, R., Farber, S., Grasso, M., Hanonnon, B., Limburg, K., Naeem, S., O'Neill, R., Paruelo, J., Raskin, R.G. et P. Sutton. 1997. The value of the world's ecosystem services and natural capital. Nature 387: 253-260.

David, G., Herrenschmidt, J.-B. and E. Mirault. 2007. Valeur sociale et économique des récifs coralliens du Pacifique insulaire / Social and economic values of pacific coral reefs. Coral Reef Initiatives for the Pacific (CRISP). Nouméa: South Pacific Commission.

Dirlik, A. 1996. The past as legacy and project. American Indian Culture and Research Journal 20(2): 131.

Doohan, K. 2008. Making things come good: relations between Aborigines and miners at Argyle. Western Australia: Backroom Press.

Doumenge, F. 2003. La France confrontée au 'trou noir' du Pacifique, la face inconnue de la question calédonienne. Conflits actuels, Revue d'étude politique 10: 101-112.

Doumenge, J.-P. 2000. L'outre-mer français. Paris: Armand Colin.

Doumenge, J.-P. 2002. Pluriculturalité, «ville » et «brousse » en Nouvelle-Calédonie, Communication au colloque sur l'État pluriculturel, Nouméa, Université de la Nouvelle-Calédonie, July. 11 pp.

Freyss, J. 1995. Économie assistée et changement social en Nouvelle-Calédonie. Paris: Tiers Monde, I.E.D.ES.

Gay, J.-C. 2003. L'outre-mer français - un espace singulier. Paris: Editions Belin.

Guiart, J. 1999. Les Mélanésiens devant l'économie de marché. Nouméa: Le Rocher-à-la-Voile.

Harvey, D. 2000. Spaces of hope. Edinburgh: Edinburgh University Press.

Hawke, S. et M. Gallagher. 1989. Noonkanbah. Fremantle: Fremantle Arts Centre.

Herrenschmidt, J.-B. 2003. Territorialité et identités en Mélanésie, in Guillaud, D., Huetz de Lemps, C., et O. Sevin (eds.) Îles rêvées - territoires et identités en crise dans le Pacifique insulaire. Paris: Presses de l'Université de Paris-Sorbonne, publié avec la collaboration de l'IRD. Pp291-324.

Herrenschmidt, J.-B. 2004. Territoires coutumiers et projets de développement en Mélanésie du Sud (Îles Loyauté, Vanuatu, Fidji). Thèse de Doctorat de Géographie. Paris: Université Paris IV - Sorbonne.

Horowitz, L.S. 2009. Environmental violence and crises of legitimacy in New Caledonia. Political Geography 28: 248-258.

Horowitz, L.S. 2003. Stranger in one's own home: a micropolitical analysis of the engagements of Kanak villagers with a multinational mining project in New Caledonia. PhD thesis. Canberra: Australian National University. http://hdl.handle.net/1885/47218

Houchot, M.A. 2003. Espace géographique et territoire de pêche. Etude appliquée à la tribu mélanésienne d'Oundjo, Nouvelle-Calédonie. Mémoire de maîtrise en géographie. Papeete: Université de la Polynésie Française.

Kowasch, M. 2010. Les populations kanak face au développement de l'industrie du nickel en NouvelleCalédonie. Thèse de Doctorat de Géographie, Université Montpellier III/ Université de Heidelberg. http://www.ub.uni-heidelberg.de/archiv/12305

Kowasch, M. 2012. La zone Voh-Koné-Pouembout. In: J. Banvallot and J.-Ch. Gay, Atlas de la NouvelleCalédonie. Montpellier: IRD Editions. Pp13-16.

Le Meur, P.-Y. 2010a. Réflexions sur un oxymore - Le débat du " cadastre coutumier » en NouvelleCalédonie. In Faugère, E. et Merle, I. (eds.) La Nouvelle-Calédonie, vers un destin commun? Paris: Editions Karthala. Pp 101-127.

Le Meur, P.-Y. 2010b. La terre en Nouvelle-Calédonie: pollution, appartenance et propriété intellectuelle. Multitudes 41: 91-98.

Massey, D. 1999. Power-geometries and the politics of space-time. Franz Steiner Verlag.

Moberg, F. et C. Folke. 1999. Ecological goods and services of coral reef ecosystems. Ecological Economics 29: 215-233.

Naepels, M. 1998. Histoires de terres kanak. Paris: Editions Belin.

Naepels, M. 2006. Réforme foncière et propriété dans la région de Houaïlou (Nouvelle-Calédonie). Etudes rurales Janvier-Juin: 43-54.

Néaoutyine, P. 2006. l'Indépendance au présent - identité Kanak et destin commun. Paris: Editions Syllepse.

Parry, B. 2002. Cultures of knowledge: investigating intellectual property rights and relations in the Pacific. Antipode 34(4): 679-707.

Perret, C. 2002.Perpectives de développement pour la Nouvelle-Calédonie. Presses Universitaires de Grenoble.

Pitoiset, A. et C. Wéry. 2008. Mystère Dang. Nouméa: Le Rayon Vert.

Speed, S. 2002. Global discourses on the local terrain. Cultural Dynamics 14 (2): 205-228.

Watts, M.J. 1999. Collective wish images: geographical imaginaries and the crisis of development. In J. Allen \& D. Massey (Eds.). Human geography today. Cambridge University Press. Pp 85-107. 
Watts, M.J. 2001. Petro-violence: nation, identity and extraction in Nigeria and Ecuador. In N. Peluso \& M.J. Watts (Eds.) Violent environments. Ithaca: Cornell University Press.

Winslow, D. 1995. Indépendance, savoir aborigène et environnement en Nouvelle-Calédonie. Journal of Political Ecology 2: 1-19.

\begin{abstract}
New Caledonia is characterized by cultural diversity, and human occupation of the territory is divided. A Melanesian, Kanak agrarian society (about $40 \%$ of the total population), and a largely urban society, of European and other origins (about 60\%), co-inhabit a territory of approximately $19,000 \mathrm{~km}^{2}$. The duality of occupation is also shown in the juxtaposition of common and customary land laws. These are the result of a painful history of land dispossession during colonial times and restitution of some land to the Kanak from 1970. Kanak identity is built on the clan's history inscribed in a natural milieu where the environment, and land, has customary value, more than use value. New Caledonia has considerable mineral resources, especially nickel. Mining often creates conflict, as it raises the use value of land. Therefore, the establishment of a mine, refinery or industrial zone can often initiate assertions of clan ownership and land claims. Land rights are constantly updated, and can be renegotiated. The remodeling of the territory under mining pressures and new land allocations is a means for upward social mobility and prestige in Kanak society. These issues are demonstrated for the Federation "Djelawe" and two tribes (Oundjo and Baco) near the site of the future nickel ore processing plant and port (the Koniambo project) in the north of Grande Terre built by the local SMSP company and the Swiss Xstrata group. A discourse of environmental protection was used to restrain industrial activity but also to assert rights to clan land. But development pressures have also been used to achieve political control over land, and thus to increase clan recognition, and possible royalty payments. Thus, land claims are part of a game of prestige and power between clans and families. Socio-economic access to land, it emerges, is clearly more important in these cases than the protection of its bio-physical assets.
\end{abstract}

Key words: New Caledonia, Kanak, land conflicts, nickel mining, regional development.

\title{
Résumé
}

La Nouvelle-Calédonie se caractérise par une grande diversité culturelle, mais également par une dualité des espaces de vie. Une société agraire multiséculaire, d'origine kanak (environ $40 \%$ de la population totale), et une société majoritairement urbaine, d'origine européenne, mais largement métissée (environ $60 \%$ de la population totale), co-habitent sur un territoire d'environ $19,000 \mathrm{~km}^{2}$ qui possèdent des ressources minérales considérables, surtout en nickel. La dualité des espaces de vie se montre également dans la juxtaposition de terres soumises au droit commun et de terres soumises au droit coutumier. Ces dernières sont le fruit d'une histoire douloureuse de spoliations foncières lors de l'époque coloniale et de rétrocessions à partir des terres 1970. La perception territoriale de la population kanak s'oriente vers un modèle où la valeur patrimoniale prime sur la valeur d'usage, car l'identité kanak se construit sur l'histoire du groupe inscrit dans un environnement où tous les objets environnementaux possèdent une certaine valeur. La co-existence des lieux à forte valeur patrimoniale, les lieux sacrés, et une activité minière ou économique au sens large peut entraîner une transformation de la valeur et suscite souvent des conflits, car une légitimité foncière signifie un plus de prestige. De ce fait, la mise en place d'un projet économique - c'est-à-dire une mine, une usine métallurgique ou une zone industrielle - réveille souvent des revendications foncières. Ces revendications démontrent que les légitimités foncières sont en perpétuelle réactualisation et peuvent être renégociées. Le remodelage du territoire représente un moyen pour une ascension sociale au sein de la société kanak. Ces enjeux fonciers sont démontrés à l'exemple de la fédération «Djelawe » et de deux tribus (Oundjo et Baco) en proximité du site industriel de la future « usine du Nord », construite par un consortium de la SMSP locale et du groupe suisse Xstrata (projet Koniambo). Depuis un certain temps, la protection de l'environnement devient une préoccupation de plus en plus importante des acteurs locaux. Ce discours environnementaliste est cependant souvent instrumentalisé pour atteindre des objectifs "politico-fonciers »: une reconnaissance foncière et des royalties. Ainsi, les revendications foncières s'inscrivent dans un jeu de prestige et de pouvoir entre clans et familles. L'aspect socio-économique de l'environnement semble être clairement plus important que l'aspect bio-physique.

Mots clés: Nouvelle-Calédonie, Kanak, les conflits fonciers, l'exploitation minière du nickel, du développement régional. 


\section{Resumen}

Nueva Caledonia se caracteriza por su gran diversidad cultural, y la ocupación humana del territorio está dividida. Una sociedad melanesia, kanaka y agrícola (aproximadamente el $40 \%$ de la población) y un sociedad mayoritariamente urbana de origen europeo y de otras zonas del mundo (aproximadamente $60 \%$ ), cohabitan un territorio de aproximadamente $19.000 \mathrm{~km}^{2}$ con considerables recursos naturales como el níquel. La dualidad de la ocupación también se observa en la yuxtaposición de tierras sometidas al derecho común y tierras sometidas al derecho tradicional. Éstas son el resultad de una historia dolorosa de expoliaciones financieras durante la época colonial y el resultado de la restitución de algunas tierras a los kanakos desde los años 70 del siglo XX. La percepción territorial de la población kanaka se inscribe dentro de un modelo donde el valor patrimonial prima por encima del valor de uso, ya que la identidad kanaka se construye sobre la historia de un grupo inscrito dentro de un marco en el que todos los elementos medioambientales poseen un cierto valor. La coexistencia de lugares de fuerte valor patrimonial, lugares sagrados y una actividad minera o económica de gran escala puede conllevar una transformación de los valores y suscitar conflictos, ya que la legitimidad territorial significa un mayor prestigio. Por tanto, el establecimiento de proyectos como una mina, una refinería o una zona industrial pueden llevar a menudo a reivindicaciones territoriales. Estas reivindicaciones demuestran que los derechos territoriales son constantemente variables. La remodelación territorial representa un medio de ascensión social en el seno de la sociedad kanaka. Estos asuntos aparecen desmostrados en el ejemplo de la federación Djelawe y en dos tribus (Oundjo y Baco) cercanas al área industrial de la futura "fábrica del norte" construida por un consorcio de la compañía SMSP local y el grupo suizo Xstrata (proyecto Koniambo). Tras un tiempo, la protección medioambiental se convierte en una preocupación cada vez mayor para los locales. Este discurso mediambiental sin embargo a menudo es instrumentalizado para alcanzar los objetivos político-territoriales: reconocimiento territorial y los royalties. Así, las reivindicaciones territoriales se inscriben dentro de un juego de prestigio y de poder entre clanes y familias. El aspecto socio-económico del medioambiente parece ser claramente más importante que el aspecto bio-físico.

Palabras clave: Nueva Caledonia, Kanakos, conflictos territoriales, minería de níquel, desarrollo regional. 